\title{
An Abel map to the compactified Picard scheme realizes Poincaré duality
}

\author{
JESSE LEO KASS \\ KIRSTEN WICKELGREN
}

For a smooth algebraic curve $X$ over a field, applying $\mathrm{H}_{1}$ to the Abel map $X \rightarrow$ Pic $X / \partial X$ to the Picard scheme of $X$ modulo its boundary realizes the Poincaré duality isomorphism

$$
\mathrm{H}_{1}(X, \mathbb{Z} / \ell) \rightarrow \mathrm{H}^{1}(X / \partial X, \mathbb{Z} / \ell(1)) \cong \mathrm{H}_{c}^{1}(X, \mathbb{Z} / \ell(1)) .
$$

We show the analogous statement for the Abel map $X / \partial X \rightarrow \overline{\text { Pic }} X / \partial X$ to the compactified Picard, or Jacobian, scheme, namely this map realizes the Poincaré duality isomorphism $\mathrm{H}_{1}(X / \partial X, \mathbb{Z} / \ell) \rightarrow \mathrm{H}^{1}(X, \mathbb{Z} / \ell(1))$. In particular, $\mathrm{H}_{1}$ of this Abel map is an isomorphism.

In proving this result, we prove some results about $\bar{P}$ ic that are of independent interest. The singular curve $X / \partial X$ has a unique singularity that is an ordinary fold point, and we describe the compactified Picard scheme of such a curve up to universal homeomorphism using a presentation scheme. We construct a MayerVietoris sequence for certain pushouts of schemes, and an isomorphism of functors $\pi_{1}^{\ell} \operatorname{Pic}^{0}(-) \cong \mathrm{H}^{1}\left(-, \mathbb{Z}_{\ell}(1)\right)$.

14F35; 14D20, 14F20

\section{Introduction}

In this paper we extend a classical relation between Poincaré duality and the Abel map. The classical Abel map of a smooth proper curve over a field $k$ is a map Ab from the curve $X$ to its Picard, or Jacobian, scheme Pic $X$. The Picard scheme Pic $X$ is the moduli space of invertible sheaves, and Ab sends a point $x$ to the sheaf $\mathcal{O}_{X}(x)$ of rational functions with at worst a pole at $x$. The Abel map induces a homomorphism from the homology of $X$ to the homology of Pic $X$. Moreover, there is a canonical isomorphism $\sigma$ between the homology of Pic $X$ and the cohomology of $X$, making the composition $\sigma^{-1} \circ \mathrm{H}_{1}(\mathrm{Ab})$ a homomorphism between the homology and the cohomology of $X$. This composition is exactly the Poincaré duality isomorphism

$$
\text { Poincaré duality }=\sigma^{-1} \circ \mathrm{H}_{1} \text { (Abel map). }
$$


In other words, the Abel map of a smooth proper curve realizes the Poincaré duality isomorphism. In this paper we extend this result by showing that the Poincaré duality isomorphism associated to a smooth nonproper curve is realized by the Abel map from an explicit singular proper curve $X^{+}$to its compactified Picard scheme. The singular curve $X^{+}$plays the role of the curve modulo its boundary, as in topological Poincaré duality of manifolds.

When $X$ is smooth and proper, the isomorphism $\sigma$ is constructed as follows. Fix a prime $\ell$ distinct from the characteristic char $k$. The étale cohomology of $X$ with coefficients in the Tate twist $\mathbb{Z} / \ell(1)=\mu_{\ell}$, or $\ell^{\text {th }}$ roots of unity, is recorded by Pic $X$ in the following manner. The points of Pic $X$ are invertible sheaves or, equivalently, $\mathbb{G}_{m}-$ torsors and thus correspond to elements of $\mathrm{H}^{1}\left(X, \mathbb{G}_{m}\right)$. The inclusion $\mathbb{Z} / \ell(1) \rightarrow \mathbb{G}_{m}$ induces an isomorphism

$$
\mathrm{H}^{1}\left(X_{\bar{k}}, \mathbb{Z} / \ell(1)\right) \stackrel{\cong}{\Rightarrow} \operatorname{Pic} X[\ell](\bar{k})
$$

from étale cohomology to the $\ell$-torsion subgroup of the group of $\bar{k}$-valued points of Pic $X$, as is seen with the long exact sequence associated to the Kummer exact sequence

$$
1 \longrightarrow \mathbb{Z} / \ell(1) \longrightarrow \mathbb{G}_{m} \stackrel{z \mapsto z^{\ell}}{\longrightarrow} \mathbb{G}_{m} \longrightarrow 1 .
$$

Thus the moduli definition of the Picard scheme identifies its torsion points with $\mathrm{H}^{1}\left(X_{\bar{k}}, \mathbb{Z} / \ell(1)\right)$.

The structure of the Picard scheme computes these torsion points in terms of its fundamental group. Let $\operatorname{Pic}^{0} X$ denote the connected component of Pic $X$ containing the identity $e$, and note that the open immersion $\operatorname{Pic}^{0} X \rightarrow \operatorname{Pic} X$ determines a canonical isomorphism $\pi_{1}\left(\mathrm{Pic}^{0} X_{\bar{k}}, e\right) \rightarrow \pi_{1}\left(\operatorname{Pic} X_{\bar{k}}, e\right)$ between the fundamental group of $\mathrm{Pic}^{0} X_{\bar{k}}$ based at the identity and the fundamental group of Pic $X_{\bar{k}}$ based at the identity. The multiplication-by- $\ell$ map $\operatorname{Pic}^{0} X \rightarrow \operatorname{Pic}^{0} X$, defined as $I \mapsto I^{\otimes \ell}$, is a Galois covering space map with Galois group equal to the group of $\ell$-torsion, inducing a homomorphism

$$
\pi_{1}\left(\operatorname{Pic} X_{\bar{k}}, e\right) \rightarrow \operatorname{Pic} X[\ell](\bar{k})
$$

from the fundamental group of Pic $X_{\bar{k}}$ to Pic $X[\ell](\bar{k})$. The Serre-Lang theorem implies that (3) becomes an isomorphism after tensoring $\pi_{1}\left(\operatorname{Pic} X_{\bar{k}}, e\right)$ with $\mathbb{Z} / \ell$. Since the fundamental group is abelian, for instance by Serre-Lang or alternatively by the Eckmann-Hilton argument, for any definition of homology satisfying the Hurewicz theorem, there is an isomorphism

$$
\mathrm{H}_{1}\left(\operatorname{Pic} X_{\bar{k}}, \mathbb{Z} / \ell\right) \stackrel{\cong}{ } \operatorname{Pic} X[\ell](\bar{k}) .
$$


Define $\sigma$ to be the composition of (2) with the inverse of (4). Equation (1) is then a consequence of Proposition 3.4 of SGA $4 \frac{1}{2}$ [9, Dualité, page 164].

In other words, the canonical isomorphism $\sigma$ results from combining the moduli definition of Pic with its structure theory.

In this paper we extend the relation between the classical Abel map and Poincaré duality for a smooth proper curve to a relation between the Altman-Kleiman Abel map and Poincaré duality for a smooth nonproper curve, which we place in analogy with manifolds with boundary. Topological Poincaré duality works for manifolds with boundary, resulting in isomorphisms between the homology of the manifold modulo its boundary and the cohomology of the manifold. We will discuss the algebraic analogue below, in which to a smooth nonproper algebraic curve $X$ (satisfying a technical assumption), we associate a singular curve $X^{+}$which should be thought of as $X$ modulo a sort of natural boundary.

The Altman-Kleiman Abel map does not embed $X^{+}$into its Picard scheme, but rather embeds $X^{+}$into its compactified Picard scheme. Like the Picard scheme, the compactified Picard scheme has a moduli definition and structure theory, of course, but the structure of the compactified Picard scheme is more complicated than that of the Picard scheme, and is studied in this paper.

\subsection{The main result}

This paper computes the structure of the compactified Picard scheme of $X^{+}$, and uses this computation to show that Poincaré duality for a nonproper smooth curve $X$ is realized by the Abel map of $X^{+}$, extending the result discussed above. Embedded in this statement is the claim that the compactified Picard scheme admits a canonical isomorphism

$$
\sigma: \mathrm{H}^{1}\left(X_{\bar{k}}, \mathbb{Z} / \ell(1)\right) \stackrel{\cong}{\rightrightarrows} \mathrm{H}_{1}\left(\overline{\mathrm{Pic}} X_{\bar{k}}^{+}, \mathbb{Z} / \ell\right) .
$$

We develop a structure theory for $\bar{P}$ ic $X^{+}$which, when combined with the moduli definition of Pic, allows us to define $\sigma$.

It is natural to consider a smooth curve modulo its boundary in the context of Poincaré duality. The Poincaré duality perfect pairing

$$
\mathrm{H}^{i}(M / \partial M, \mathbb{Z} / \ell) \otimes \mathrm{H}^{\operatorname{dim} M-i}(M, \mathbb{Z} / \ell) \rightarrow \mathbb{Z} / \ell
$$

for an oriented manifold $M$ with boundary $\partial M$ follows from Michael Atiyah's formula [5] for the dual of $M / \partial M$ in terms of the tangent bundle of $M$ in the category of spectra. In this sense, $M / \partial M$ is dual to a shift of $M$ itself. The analogous duality in algebraic geometry, or rather $\mathbb{P}^{1}$-spectra (see Hu [19]), produces perfect pairings of 
étale cohomology groups; see Isaksen [20]. In particular, let $X$ be a smooth curve and assume that $X$ is an open subscheme of a smooth proper curve $\tilde{X}$ such that the residue fields of the points of $\tilde{X}-X$ are separable extensions of $k$. (For instance, when $k$ has characteristic 0 this assumption is automatically satisfied.) Form $X / \partial X:=X^{+}$, where $X^{+}$is defined by the pushout diagram (see Ferrand [15, Theorem 5.4])

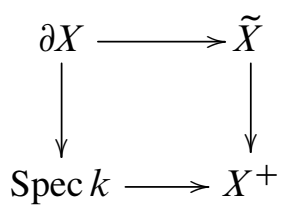

and $\partial X$ denotes the complement $\tilde{X}-X$ with its reduced closed subscheme structure. (This pushout is discussed further in Section 2.) Let $R$ denote $\mathbb{Z} / \ell$ or $\mathbb{Z}_{\ell}$, with $\ell$ relatively prime to char $k$. By Milne [25, Corollary 11.2, Chapter VI] and the canonical isomorphism $\mathrm{H}_{c}^{1}\left(X_{\bar{k}}, R(r)\right) \cong \mathrm{H}^{1}\left(X_{\bar{k}}^{+}, R(r)\right)$, there is a perfect pairing of étale cohomology groups

$$
\mathrm{H}^{1}\left(X_{\bar{k}}^{+}, R(r)\right) \otimes \mathrm{H}^{1}\left(X_{\bar{k}}, R(1-r)\right) \rightarrow R .
$$

For simplicity, define $\mathrm{H}_{1}\left((-)_{\bar{k}}, R\right)$ for geometrically connected $k$-schemes using the abelianization of the $\ell$-étale fundamental group by

$$
\mathrm{H}_{1}\left((-)_{\bar{k}}, R\right):=\pi_{1}^{\ell}(-)_{\bar{k}}^{\mathrm{ab}} \otimes_{\mathbb{Z}_{\ell}} R,
$$

but see Friedlander [16, Section 7] for a discussion of étale homology in more generality. The tautological pairing between $\mathrm{H}_{1}$ and $\mathrm{H}^{1}$ produces the Poincaré duality isomorphisms (see Section 7)

$$
\begin{aligned}
& \text { Poincaré duality: } \mathrm{H}_{1}\left(X_{\bar{k}}, R\right) \rightarrow \mathrm{H}^{1}\left(X_{\bar{k}}^{+}, R(1)\right), \\
& \text { Poincaré duality: } \mathrm{H}_{1}\left(X_{\bar{k}}^{+}, R\right) \rightarrow \mathrm{H}^{1}\left(X_{\bar{k}}, R(1)\right) .
\end{aligned}
$$

It is not hard to modify the argument given in the proper case to show that an Abel map realizes the isomorphism (5). More explicitly, the moduli space Pic $X^{+}$of invertible sheaves on $X^{+}$exists, and the rule $x \mapsto \mathcal{O}_{X^{+}}(x)$ defines an Abel map

$$
\text { Ab: } X \rightarrow \operatorname{Pic} X^{+}
$$

whose domain is the nonproper curve $X$. The connected component of Pic $X^{+}$ containing the trivial line bundle is a semi-abelian variety, and a generalization of the Serre-Lang theorem due to Mochizuki [26, Appendix, page 221 (III)] computes the fundamental group of Pic $X^{+}$in terms of torsion points. Using the fact that these torsion points are line bundles, a canonical isomorphism $\sigma$ can be constructed as before, and this isomorphism satisfies (1). 
What about the isomorphism (6)? The rule $x \mapsto \mathcal{O}_{X}(x)$ does not define an Abel map from $X^{+}$to Pic $X^{+}$because $\mathcal{O}_{X}(x)$ is undefined when $x$ is a singularity. The generalized Picard scheme Pic $X^{+}$is not proper, but it is contained in the proper scheme $\bar{M}$ od $X^{+}$defined as the moduli space of rank-1, torsion-free sheaves on $X^{+}$. The ideal sheaf $I_{X}=\mathcal{O}_{X^{+}}(-x)$ of a point on $X^{+}$is a rank-1, torsion-free sheaf, and so the rule $x \mapsto I_{x}$ defines a map $X^{+} \rightarrow \overline{\operatorname{M}}$ od $X^{+}$. The scheme $\overline{\operatorname{M}}$ od $X^{+}$has the undesirable property that the connected components are reducible, so rather than working with $\bar{M}$ od $X^{+}$we work with the Zariski closure of the line bundle locus, which we call the compactified Picard scheme $\bar{P}$ ic $X^{+}$. The rule $x \mapsto I_{x}$ defines a morphism Ab: $X^{+} \rightarrow \overline{\text { Pic }} X^{+}$, which will be called the Abel map of $X^{+}$.

The main theorem of this paper is that Ab realizes the Poincaré duality isomorphism (6). The theorem applies under the following hypothesis:

Hypothesis 1.2 The curve $X$ has the following properties:

(1) The normal proper model $\tilde{X}$ of $X$ is smooth over $k$.

(2) The extension of residue fields $k(x) / k$ is separable for every point

$$
x \in \partial X:=\tilde{X}-X \text {. }
$$

The theorem states the following:

Theorem 1.3 If $X$ is a smooth curve over $k$ that satisfies Hypothesis 1.2, then

$$
\text { -Poincaré duality }=\sigma^{-1} \circ \mathrm{H}_{1} \text { (Abel map) }
$$

for an explicit isomorphism of $\mathrm{Gal}(\bar{k} / k)$-modules $\sigma: \mathrm{H}^{1}\left(X_{\bar{k}}, R(1)\right) \rightarrow \mathrm{H}_{1}\left(\overline{\mathrm{P}} \mathrm{ic} X_{\bar{k}}^{+}, R\right)$.

This result is Theorem 7.1 below. Observe that in (7), Poincare duality appears with a minus sign. We discuss the significance of this sign and Hypothesis 1.2 later in this introduction.

We prove Theorem 1.3 using a strategy similar to the one used to prove (1). The isomorphism $\sigma$ is constructed using a description of the structure of $\overline{\text { Pic }} X^{+}$and the definition of Pic $\tilde{X}$ as a moduli space. We then prove (7) using analogues of results of SGA $4 \frac{1}{2}$ [9, Dualité, Section 3]; we also obtain a generalization of Propositions 3.2 and 3.4 therein in the case of a proper smooth curve $\tilde{X}$. Together, these two propositions imply that the Poincare dual of a loop on $\tilde{X}$ can be described as the associated monodromy on the pullback of the multiplication-by- $\ell$ map Pic $\rightarrow$ Pic under a translation of the Abel map. We give the monodromy of a path in terms of Poincaré duality on a quotient curve. The different fibers have a canonical identification after tensoring with the cohomology of the quotient curve, allowing us to speak of monodromy as an element of this cohomology. See Section 7. 


\subsection{New results about $\bar{P} i c X^{+}$}

In proving Theorem 1.3, we prove new results about the compactified Picard scheme that are of independent interest. Unlike the Picard scheme of a smooth proper curve, the compactified Picard scheme does not have a group structure, so we cannot use, for example, the Serre-Lang theorem, and instead compute the structure of Pic $X^{+}$ up to universal homeomorphism. Prior to this paper, very little was known about $\overline{\mathrm{Pic}} X^{+}$when $\partial X$ contains at least three points. In this case, the singularity of $X^{+}$is non-Gorenstein and non-planar. While there is a large body of work describing the structure of the compactified Picard scheme of a curve with planar singularities, the only results that apply to $X^{+}$that the authors are aware of are Altman and Kleiman's result that $\overline{\mathrm{P}} \mathrm{ic} X^{+}$is a projective scheme [1] and Kleiman and Kleppe's result that the moduli space $\overline{\operatorname{Mod}}^{d} X^{+}$of degree- $d$, rank-1, torsion-free sheaves is reducible [23].

To construct $\sigma$ in Theorem 1.3, we describe the structure of $\bar{P}$ ic $X^{+}$as follows. Let $f: \tilde{X} \rightarrow X^{+}$denote the map from the pushout definition of $X^{+}$, which is also the normalization map. Let $x_{0}$ denote the singular point of $X^{+}$. There is a projective bundle Pres $X^{+}$over the Picard scheme $\mathrm{Pic}^{-1} \tilde{X}$ representing a certain presentation functor, and a birational morphism Pres $X^{+} \rightarrow \overline{\mathrm{Pic}}^{-1} X^{+}$from the bundle to the compactified Picard scheme. The bundle map Pres $X^{+} \rightarrow \mathrm{Pic}^{-1} \tilde{X}$ admits sections labeled by the points of $f^{-1}\left(x_{0}\right)$, and the birational morphism Pres $X^{+} \rightarrow \bar{P}^{-1} X^{+}$ is the map that, up to universal homeomorphism, identifies these sections in the sense that a natural pushout diagram (21) defines a universal homeomorphism. This is Theorem 3.19.

The geometric description of $\bar{P}^{-1} X^{+}$given by Theorem 3.19 is of independent interest. For example, the theorem (or more precisely Proposition 3.16, which is used to prove the theorem) shows that a rank-1, torsion-free sheaf lies in $\overline{\mathrm{Pic}}{ }^{-1} X^{+}$, rather than in a different component of the reducible scheme $\overline{\operatorname{Mod}}{ }^{-1} X^{+}$, precisely when the sheaf is the direct image of a line bundle under a partial normalization map $\bar{Y} \rightarrow X^{+}$ out of a curve $\bar{Y}$ with at most one singularity. In particular, we have:

Corollary 1.5 Assume char $k>3$. Define $X^{+}$to be the rational curve with a 4fold point that is obtained from $\tilde{X}:=\mathbb{P}^{1}$ by identifying the points $\partial X=\{ \pm 1, \pm 2\}$. Define $\bar{Y}$ to be the rational curve with two nodes that is obtained from $\tilde{X}$ by identifying 1 with -1 and 2 with -2 . If $g: \bar{Y} \rightarrow X^{+}$is the natural morphism, then

$$
I=g_{*} \mathcal{O}_{\bar{Y}}
$$

is not the limit of line bundles. 
Note that when $\partial X$ consists of two points, the singularity of $X^{+}$is a node. The projective bundle Pres $X^{+}$that appears in Theorem 3.19 is a generalization of the presentation scheme of a nodal curve constructed by Oda and Seshadri [29] and Altman and Kleiman [2] and as such, is also called the presentation scheme. The presentation scheme of a nodal curve was also studied by Bhosle in [6], where the scheme appears as an example of a moduli space of generalized parabolic bundles.

Let us use what has been said about the structure of $\overline{\mathrm{Pic}}^{-1} X^{+}$to define $\sigma$. Assume for simplicity that $k=\bar{k}$, and that $\tilde{X}$ has genus greater than 0 . As above, we have the presentation scheme Pres $X^{+}$and maps

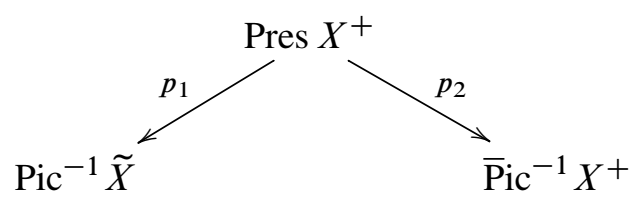

with $p_{1}$ a projective bundle, equipped with sections in bijection with the points of $\partial X$, or equivalently in bijection with the points of $f^{-1}\left(x_{0}\right)$, and $p_{2}$ a quotient map identifying the images of the sections in a certain manner. As $\tilde{x}$ varies over all points of $\partial X$, apply the corresponding section to $\operatorname{Ab}(\tilde{x})$ to produce a set $\mathcal{E}$ of points of Pres $X^{+}$. In the description of $p_{2}$, we will see that the points of $\mathcal{E}$ all have the same image under $p_{2}$, inducing a map from the étale fundamental groupoid $\pi_{1}$ (Pres $X^{+}, \mathcal{E}$ ) to the étale fundamental group of $\overline{\mathrm{Pic}}^{-1} X^{+}$. Composing with the Hurewicz map, which is tautological with our definition of $\mathrm{H}_{1}$, yields

$$
\pi_{1}^{\ell}\left(\operatorname{Pres} X_{\bar{k}}^{+}, \mathcal{E}\right) \rightarrow \mathrm{H}_{1}\left(\overline{\mathrm{Pic}}^{-1} X_{\bar{k}}^{+}, R\right) .
$$

The projective bundle $p_{1}$ identifies this fundamental groupoid with the fundamental groupoid of $\mathrm{Pic}^{-1} \tilde{X}$ based at the images of the points of $\partial X$ under the Abel map

$$
p_{1 *}: \pi_{1}^{\ell}\left(\operatorname{Pres} X^{+}, \mathcal{E}\right) \stackrel{\cong}{\rightrightarrows} \pi_{1}^{\ell}\left(\operatorname{Pic}^{-1} \tilde{X}, \mathrm{Ab}_{*}\left(\partial X_{\bar{k}}\right)\right) \text {. }
$$

In Theorem 5.1, we show that the moduli definition of Pic yields a canonical isomorphism

$$
\mathcal{F}_{R} \pi_{1}^{\ell}\left(\mathrm{Pic}^{-1} \tilde{X}, \mathrm{Ab}_{*}\left(\partial X_{\bar{k}}\right)\right) \rightarrow \mathrm{H}^{1}\left(X_{\bar{k}}, R(1)\right)
$$

from the free $R$-module $\mathcal{F}_{R} \pi_{1}^{\ell}\left(\operatorname{Pic}^{-1} \tilde{X}_{\bar{k}}, \mathrm{Ab}_{*}\left(\partial X_{\bar{k}}\right)\right)$ on the fundamental groupoid

$$
\pi_{1}^{\ell}\left(\operatorname{Pic}^{-1} \tilde{X}_{\bar{k}}, \mathrm{Ab}_{*}\left(\partial X_{\bar{k}}\right)\right)
$$

to the étale cohomology group $\mathrm{H}^{1}\left(X_{\bar{k}}, R(1)\right)$.

The map (8) and universal property of $\mathcal{F}_{R}$ then give

$$
\sigma: \mathrm{H}^{1}\left(X_{\bar{k}}, R(1)\right) \rightarrow \mathrm{H}_{1}\left(\overline{\mathrm{Pic}}_{\bar{k}}^{+}, R\right)
$$


which is shown to be an isomorphism in Proposition 6.1. This is the $\sigma$ which appears in Theorem 1.3.

\subsection{Connections with autoduality}

Theorem 1.3 is related to the theory of autoduality of the compactified Picard scheme. Under various hypotheses on $\bar{X}$, Arinkin [4], Esteves, Gagné and Kleiman [13], Esteves and Rocha [14], and Melo, Rapagnetta and Viviani [24] have proved that Pic(Ab) is an isomorphism between the Picard schemes $\operatorname{Pic}^{0} \bar{X}$ and $\operatorname{Pic}^{0} \bar{P} i c \bar{X}$. How are those results related to the results of this paper?

One consequence of Theorem 1.3 is the following.

Corollary 1.7 Applying either the functor $\mathrm{H}_{1}\left((-)_{\bar{k}}, R\right)$ or $\mathrm{H}^{1}\left((-)_{\bar{k}}, R\right)$ to

$$
\mathrm{Ab}: X^{+} \rightarrow \overline{\mathrm{Pic}} X^{+}
$$

produces an isomorphism.

For cohomology, the result is established using a tautological pairing between

$$
\mathrm{H}_{1}\left((-)_{\bar{k}}, R\right) \text { and } \mathrm{H}^{1}\left((-)_{\bar{k}}, R\right),
$$

discussed in the second paragraph of Section 7 and Appendix B (41). The pairing is induced from the monodromy pairing between torsors and $\pi_{1}$.

Results similar to Corollary 1.7 can be deduced from autoduality results using the following proposition:

Proposition 1.8 Let $k$ be a perfect field. There is a natural isomorphism of functors from proper, geometrically connected schemes over $k$ to $\mathrm{Gal}(\bar{k} / k)$-modules

$$
\pi_{1}^{\ell}\left(\operatorname{Pic}^{0}(-)_{\bar{k}}, e\right) \cong \mathrm{H}^{1}\left((-)_{\bar{k}}, \mathbb{Z}_{\ell}(1)\right) .
$$

We provide a proof of Proposition 1.8 in Appendix A.

The above proposition implies that Corollary 1.7 remains valid when $X^{+}$is replaced by a curve $\bar{X}$ whose compactified Picard scheme satisfies autoduality. Currently, autoduality results have only been proven under the hypothesis that $\bar{X}$ has plane curve singularities, and $X^{+}$has plane curve singularities exactly when $x_{0} \in X^{+}$is a node. For such an $X^{+}$, Theorem 2.1 of Esteves, Gagné and Kleiman [13] implies that $\mathrm{Ab}$ induces an isomorphism on homology and cohomology; alternatively, see Arinkin [3, Theorem C]. 


\subsection{Concluding remarks}

Let us conclude our discussion with two remarks about Theorem 1.3. First, in the theorem, we assume $X$ satisfies Hypothesis 1.2. This assumption allows us to assert that the curve $X_{\bar{k}}^{+}$obtained by extending scalars to $\bar{k}$ is obtained from a smooth curve by a suitable pushout, and, in particular, allows us to avoid curves that are normal but not geometrically normal (ie not smooth). Second, the conclusion of Theorem 1.3 differs from (1) by the minus sign of (7), because the Abel map of (7) is not the classical Abel map appearing in (1). Classically, the Abel map is defined by $x \mapsto \mathcal{O}_{X}(x)$, while the Abel map in (7) is defined by $x \mapsto I_{X}=\mathcal{O}_{X}(-x)$. On a smooth proper curve, the difference is a matter of notation, as they differ by the automorphism $I \mapsto \operatorname{Hom}\left(I, \mathcal{O}_{X}\right)$. On such a singular curve $X^{+}$, however, the difference is significant, as only the second rule necessarily defines a regular map, as we show in Example 4.1.

This paper is organized as follows. In Section 2 we record the definition of the one-point compactification $X^{+}$of a suitable smooth curve $X$ over $k$. We study the compactified Picard scheme $\bar{P}$ ic $X^{+}$of $X^{+}$in the next two sections. In Section 3 we define the presentation scheme and then use it in Theorem 3.19 to compute the compactified Picard scheme up to universal homeomorphism. We study the Abel map in Section 4, where we prove that the classical Abel map can fail to extend to a morphism out of $X^{+}$and that the Altman-Kleiman Abel map lifts to a morphism into the presentation scheme.

In the last three sections, we describe the homology of $\overline{\mathrm{Pic}}^{-1} X^{+}$and apply that description to prove Theorem 1.3. In Section 5 we prove that the cohomology of the smooth curve $X$ can be recovered from the fundamental groupoid of $\mathrm{Pic}^{-1} \tilde{X}$. We use this fact in Section 6 to construct the isomorphism $\sigma$ appearing in (7). We prove that (7) holds in Section 7. There are two appendices. Appendix A proves Proposition 1.8, identifying cohomology and the fundamental group of the Picard scheme. Appendix B proves a Mayer-Vietoris sequence for pushouts by closed immersions and finite maps. This sequence is of cohomology groups, or homology groups in small dimensions. The homology sequence is used in Sections 6 and 7.

\section{Conventions}

- $k$ is a field.

- $\bar{k}$ is a fixed algebraic closure of $k$.

- A curve $X / k$ is a separated, finite type, geometrically integral $k$-scheme of pure dimension 1 . 
- If $T$ is a $k$-scheme, then a family of rank-1, torsion-free sheaves on a curve $X$ parametrized by $T$ is a $\mathcal{O}_{T}$-flat finitely presented $\mathcal{O}_{X_{T}}$-module $I$ with the property that the restriction to any fiber of $X_{T} \rightarrow T$ is rank-1 and torsion-free.

- The degree-d compactified Picard scheme $\overline{\mathrm{Pic}}^{d}(\bar{X})$ of a proper curve $\bar{X}$ is the Zariski closure of the line bundle locus in the moduli space of rank-1, torsion-free sheaves of degree $d$.

- A $T$-relative effective Cartier divisor is a $T$-flat closed subscheme $D \subset X_{T}$ whose ideal $I_{D}$ is invertible.

- If $D$ is a $T$-relative effective Cartier divisor, then we write $\mathcal{O}_{X_{T}}(D)$ for $\operatorname{Hom}\left(I_{D}, \mathcal{O}_{X}\right)$.

- $\pi_{1}^{\ell}$ denotes the maximal pro- $\ell$ quotient of the étale fundamental group, and $\pi_{1}^{\left(p^{\prime}\right)}$ denotes the maximal prime-to- $p$ quotient of the étale fundamental group, where a profinite group is said to be prime-to- $p$ if it can be expressed as an inverse limit of finite groups whose orders are not divisible by $p$. If two geometric points $a$ and $b$ are included in the notation, as in $\pi_{1}(-, a, b)$, this $\pi_{1}(-, a, b)$ denotes the set of étale paths from $a$ to $b$, ie the natural transformations from the fiber functor associated to $a$ to the fiber functor associated to $b$. If $\mathcal{E}$ is a set of geometric points, $\pi_{1}(-, \mathcal{E})$ denotes the fundamental groupoid based at $\mathcal{E}$.

- $R$ denotes $\mathbb{Z}_{\ell}$ or $\mathbb{Z} / \ell^{n}$.

Acknowledgements We wish to thank Dennis Gaitsgory and Carl Mautner for useful discussions. We thank Karl Schwede for explaining seminormality to us, and Anton Geraschenko for helpful information about coproducts of schemes. We also thank Alastair King for informing us of the work of Bhosle, and Nicola Pagani, Emily Riehl, and Nicola Tarasca for helpful feedback concerning exposition.

During the writing of this paper, the first author was a Wissenschaftlicher Mitarbeiter at the Institut für Algebraische Geometrie, Leibniz Universität Hannover. The first author was supported by an AMS-Simons Travel Grant, and the second author is supported by an American Institute of Mathematics 5-year fellowship.

\section{Construction of a one-point compactification}

Here we define the one-point compactification $X^{+}$of a nonproper smooth curve $X$ over $k$. We describe the structure on the compactified Picard scheme of $X^{+}$in Section 3 below. For the remainder of this section, we fix a smooth and proper curve $\tilde{X}$ over $k$ and a collection $\partial X \subset \tilde{X}$ of closed points with the property that $k(x)$ is a separable extension $k$ for all $x \in \partial X$. We will consider $\partial X$ as a closed subscheme 
$j: \partial X \rightarrow \tilde{X}$ with the reduced scheme structure, and the separability assumption is equivalent to the assumption that $\partial X$ is $k$-étale.

To $\tilde{X}$ we associate the curve $X^{+}$defined by the pushout diagram

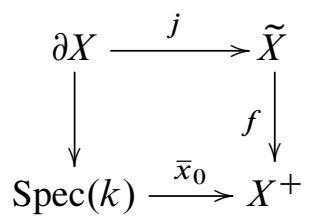

The pushout exists by [15, Theorem 5.4]. (Certainly $\tilde{X}$ satisfies Condition AF because the curve is projective.) Furthermore, $\tilde{X}$ is smooth over $k$ and $f$ is finite by [15, Proposition 5.6], so $f: \tilde{X} \rightarrow X^{+}$is the normalization map. We call the singularity $x_{0}:=\bar{x}_{0}(0)$ an ordinary fold singularity. We write $b\left(x_{0}\right):=\operatorname{rank}_{k} \mathrm{H}^{0}\left(\partial X, \mathcal{O}_{\partial X}\right)$ for the number of geometric branches of $X$ at $x_{0}$.

Diagram (9) remains a pushout diagram after tensoring with an arbitrary field extension $k^{\prime}$ of $k$ by [15, Lemma 4.4]. Since $\tilde{X}_{k^{\prime}}$ is $k^{\prime}$-smooth, $X_{k^{\prime}}^{+}$is thus constructed from a $k^{\prime}$-smooth curve by identifying a finite collection of closed points with separable residue fields.

For later use, we need a concrete description of the local ring of $X^{+}$at $x_{0}$. Ferrand constructs $X^{+}$as the pushout in the category of ringed spaces. As such a pushout, the structure sheaf $\mathcal{O}_{X^{+}}$is the pullback defined by this diagram:

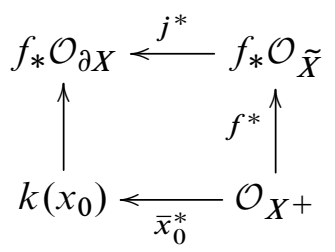

Equivalently, $\mathcal{O}_{X}+$ can be described by the exact sequence

$$
0 \rightarrow \mathcal{O}_{X^{+}} \rightarrow f_{*} \mathcal{O}_{\tilde{X}} \rightarrow f_{*} \mathcal{O}_{\partial X} / k\left(x_{0}\right) \rightarrow 0 .
$$

Taking the stalk at $x_{0}$, we get

$$
0 \rightarrow \mathcal{O}_{X^{+}, x_{0}} \rightarrow \mathcal{O}_{\tilde{X} f^{-1}\left(x_{0}\right)} \rightarrow \bigoplus_{f(\tilde{x})=x_{0}} k(\tilde{x}) / k\left(x_{0}\right) \rightarrow 0
$$

Here $\mathcal{O}_{\tilde{X} f_{-1}^{-1}\left(x_{0}\right)}$ is the semilocalization of $\tilde{X}$ at the closed subset $f^{-1}\left(x_{0}\right) \subset \tilde{X}$. When $k=\bar{k}$, we have $k(\tilde{x})=k$ for all $\tilde{x} \in f^{-1}\left(x_{0}\right)$, and so $\bigoplus k(\tilde{x}) / k\left(x_{0}\right)$ is just a $k\left(x_{0}\right)$-module of rank $b\left(x_{0}\right)-1$. 
We will occasionally need to describe the curves $\bar{Y}$ lying between $X^{+}$and its normalization $\tilde{X}$. These curves are exactly the curves constructed by partitioning the points $\partial X$ into subsets and gluing each subset together. More precisely, given a finite surjection $\partial X \rightarrow \partial Y$, the two obvious pushout squares fit into the commutative diagram:

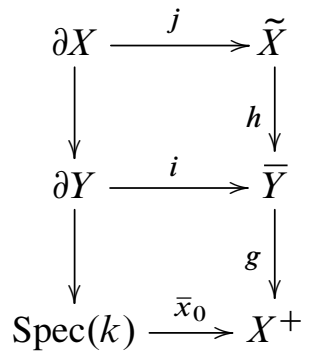

The morphism $g$ is proper and birational, so $\bar{Y}$ lies between $X^{+}$and $\tilde{X}$, and every curve lying between $X^{+}$and $\tilde{X}$ can be constructed in this manner. Indeed, suppose that we are given a factorization

$$
\tilde{X} \stackrel{h}{\longrightarrow} \bar{Y} \stackrel{g}{\longrightarrow} X^{+}
$$

with $g$ a proper birational map. The scheme $\partial Y:=g^{-1}\left(x_{0}\right)$ naturally fits into the commutative diagram (11), and now the squares are pullback squares. These squares are in fact also pushout squares. To verify this, we can reduce to the affine case (as $h$ and $g$ are affine), in which case the claim follows by direct computation.

\section{Construction of the presentation scheme}

Here we define and study the presentation scheme associated to a curve $X^{+}$from Section 2. Thus we fix a smooth curve $\tilde{X}$ over $k$ and a collection $\partial X \subset \tilde{X}$ of closed points with separable residue fields, and then define $X^{+}$by the pushout diagram (9). As in Section 2, we write $x_{0} \in X^{+}$for the unique singularity of $X^{+}, b\left(x_{0}\right):=$ $\operatorname{rank}_{k} \mathrm{H}^{0}\left(\partial X, \mathcal{O}_{\partial X}\right)$ for the number of geometric branches, and $f: \tilde{X} \rightarrow X^{+}$for the normalization of $X^{+}$.

Our definition of the presentation scheme is motivated by the following observation: if $L$ is a line bundle on $X^{+}$, then the adjoint

$$
i_{\text {can }}: L \rightarrow f_{*} f^{*} L
$$

of the identity $f^{*} L \rightarrow f^{*} L$ is an inclusion whose cokernel is naturally isomorphic to

$$
\left.f^{*} L\right|_{\partial X} /\left.L\right|_{x_{0}}=\left(\bigoplus_{f(\tilde{x})=x_{0}} k(\tilde{x}) \otimes f^{*} L\right) / k\left(x_{0}\right) \otimes L
$$


which is a $k\left(x_{0}\right)$-module of rank $b\left(x_{0}\right)-1$. This assertion is the exact sequence (10) when $L=\mathcal{O}_{X^{+}}$and is proven below in Lemma 3.12 when $L$ is any line bundle.

With $i_{\text {can }}$ in mind, we make the following definition.

Definition 3.1 Let $T$ be a $k$-scheme. A family of presentations of a family of rank-1, torsion-free sheaves $I$ over $T$ is an injective homomorphism $i: I \rightarrow\left(f_{T}\right)_{*} M$ from $I$ to the direct image of a line bundle $M$ on $\tilde{X}_{T}$ with the property that the cokernel is a locally free $\mathcal{O}_{\left\{x_{0}\right\} \times T}$-module of rank $b\left(x_{0}\right)-1$. A presentation is a family of presentations over $T=\operatorname{Spec}(k)$.

Presentations are functorial in the following sense. Suppose that we are given a morphism $s: S \rightarrow T$ and a family of presentations $i: I \rightarrow\left(f_{T}\right)_{*} M$ over $T$. Because $f$ is finite, the base-change homomorphism $\phi_{s}: s^{*}\left(f_{T}\right)_{*} M \rightarrow\left(f_{S}\right)_{*} s^{*} M$ is an isomorphism, and the resulting composition

$$
\phi_{S} \circ s^{*} i: s^{*} I \rightarrow\left(f_{S}\right)_{*} s^{*} M
$$

is a family of presentations over $S$. Indeed, because $\operatorname{Coker}(i)$ is $T$-flat, the homomorphism $s^{*} i$, or equivalently the homomorphism (14), is injective with cokernel equal to $s^{*}(\operatorname{Coker}(i))$, which is a locally free $\mathcal{O}_{\left\{x_{0}\right\} \times S}$-module of rank $b\left(x_{0}\right)-1$. We will use this functoriality to define a functor, but first we need to put an equivalence relation on presentations.

Definition 3.2 Two families of presentations $i: I \rightarrow\left(f_{T}\right)_{*} M$ and $i^{\prime}: I^{\prime} \rightarrow\left(f_{T}\right)_{*} M^{\prime}$ over $T$ are said to be equivalent if there exist a line bundle $N$ on $T$ and isomorphisms $I \cong I^{\prime} \otimes_{T} N,\left(f_{T}\right)_{*} M \cong\left(f_{T}\right)_{*} M^{\prime} \otimes N$ that make the following diagram commute:

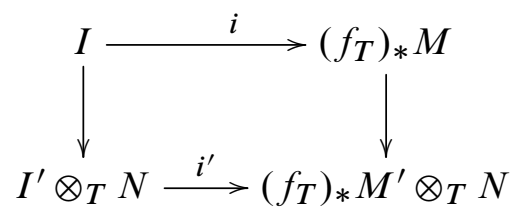

Using this definition of equivalence, we define the presentation functor.

Definition 3.3 The presentation functor Pres $^{\sharp} X^{+}$of $X^{+}$is the functor

$$
\text { Pres }{ }^{\sharp} X^{+}: k-\mathrm{Sch} \rightarrow \text { Sets }
$$

defined as follows. Given a $k$-scheme $T$, we set $\left(\operatorname{Pres}^{\#} X^{+}\right)(T)$ equal to the set of equivalence classes of families of presentations of degree -1 sheaves over $T$. Given a morphism $s: S \rightarrow T$ of $k$-schemes, we define Pres ${ }^{\sharp} s:\left(\operatorname{Pres}^{\sharp} X^{+}\right)(T) \rightarrow$ (Pres ${ }^{\sharp}\left(X^{+}\right)(S)$ by the rule that sends $i$ to the presentation $\phi_{S} \circ s^{*} i$ in (14). 
The presentation functor is represented by a Grassmannian bundle over the Picard scheme $\operatorname{Pic}^{-1} \tilde{X}$ under suitable hypotheses. Recall that the relative Grassmannian

$$
\operatorname{Grass}(\mathcal{V}, b) \rightarrow B
$$

associated to, for example, a non-negative integer $b$, an algebraic $k$-scheme $B$ and a locally free $\mathcal{O}_{B}$-module $\mathcal{V}$ is a $k$-scheme $\operatorname{Grass}(\mathcal{V}, b)$ whose $T$-valued points are pairs $(t, q)$ consisting of a $k$-morphism $t: T \rightarrow B$ and a surjective homomorphism $t^{*} \mathcal{V} \rightarrow \mathcal{W}$ onto a locally free $\mathcal{O}_{T}$-module of rank $b$.

The exact relation between a Grassmannian bundle and the presentation functor is described by the following lemma.

Lemma 3.4 Assume a universal line bundle $M_{\text {uni }}$ exists on $\tilde{X} \times \operatorname{Pic}^{-1} \tilde{X}$, and let

$$
\mathcal{V}:=\left((f \times 1)_{*}\left(M_{\text {uni }}\right)\right) \mid\left\{x_{0}\right\} \times \operatorname{Pic}^{-1} \tilde{X} .
$$

Then the presentation functor is representable by the projective $k$-scheme

$$
\operatorname{Grass}\left(\mathcal{V}, b\left(x_{0}\right)-1\right) \text {. }
$$

Remark 3.5 When $X^{+}$is a nodal curve, this lemma was proven by Altman and Kleiman [2, Proposition 9], and the following proof is closely modeled on their argument.

Proof We construct a natural transformation $\operatorname{Grass}\left(\mathcal{V}, b\left(x_{0}\right)-1\right) \rightarrow \operatorname{Pres}^{\sharp} X^{+}$, and then we construct the inverse transformation. The main point is that the cokernel of a family of presentations $i: I \rightarrow\left(f_{T}\right)_{*} M$ can be written as $q:\left(f_{T}\right)_{*} M \rightarrow\left(j_{T}\right)_{*} \mathcal{W}$ for some locally free sheaf $\mathcal{W}$ on $\left\{x_{0}\right\} \times T$ of rank $b\left(x_{0}\right)-1$, and the rule that sends $i$ to the adjoint $q^{\#}:\left(f_{T}\right)_{*} M \mid\left\{x_{0}\right\} \times T \rightarrow \mathcal{W}$ essentially defines the isomorphism $\operatorname{Pres}^{\sharp} X^{+} \cong \operatorname{Grass}\left(\mathcal{V}, b\left(x_{0}\right)-1\right)$.

We construct $\operatorname{Grass}\left(\mathcal{V}, b\left(x_{0}\right)-1\right) \rightarrow$ Pres $^{\#} X^{+}$by exhibiting a family of presentations $\operatorname{over} \operatorname{Grass}\left(\mathcal{V}, b\left(x_{0}\right)-1\right)$. Temporarily set $\mathrm{G}$ equal to $\operatorname{Grass}\left(\mathcal{V}, b\left(x_{0}\right)-1\right), g: \mathrm{G} \rightarrow$ $\left\{x_{0}\right\} \times \operatorname{Pic}^{-1} \tilde{X}$ equal to the structure morphism, $\mathcal{W}_{\mathrm{G}}$ equal to the universal quotient, $q_{\text {uni }}: g^{*} \mathcal{V} \rightarrow \mathcal{W}_{\mathrm{G}}$ equal to the universal surjection, and $j:\left\{x_{0}\right\} \rightarrow X, j^{\prime}: f^{-1}\left(x_{0}\right) \rightarrow \tilde{X}$ equal to the inclusions.

Consider the line bundle $M:=(1 \times g)^{*} M_{\text {uni }}$ on $\tilde{X} \times \mathrm{G}$. We claim that there is a canonical isomorphism $\phi_{\text {can }}:(f \times 1)_{*} M \mid \mathrm{G} \times\left\{x_{0}\right\} \cong g^{*} \mathcal{V}$. Given the claim, the composition

$$
(f \times 1)_{*} M \mid\left\{x_{0}\right\} \times \mathrm{G} \stackrel{\phi_{\mathrm{can}}}{\longrightarrow} g^{*} \mathcal{V} \stackrel{q_{\mathrm{can}}}{\longrightarrow} \mathcal{W}_{\mathrm{G}}
$$


is adjoint to a family of presentations $(f \times 1)_{*} M \rightarrow(j \times 1)_{*} \mathcal{W}_{\mathrm{G}}$ over $\mathrm{G}$, and this family defines the desired morphism $\mathrm{G} \rightarrow \operatorname{Pres}^{\#} X^{+}$.

The existence of $\phi_{\text {can }}:(f \times 1)_{*} M \mid\left\{x_{0}\right\} \times G \cong g^{*} \mathcal{V}$ follows from the cohomological flatness of $f$, which follows since $f$ is finite. Cohomological flatness implies that the base-change homomorphism

$$
(f \times 1)_{*} M \mid\left\{x_{0}\right\} \times \mathrm{G} \cong\left(\left.f\right|_{f^{-1}\left(x_{0}\right)} \times 1\right)_{*}\left(j^{\prime} \times g\right)^{*} M_{\text {uni }}
$$

is an isomorphism. A second application of cohomological flatness shows that the sheaf on the right-hand side of (15) appears in another base-change isomorphism

$$
(j \times g)^{*}(f \times 1)_{*} M_{\mathrm{uni}} \cong\left(\left.f\right|_{f^{-1}\left(x_{0}\right)} \times 1\right)_{*}\left(j^{\prime} \times g\right)^{*} M_{\mathrm{uni}},
$$

and we have

$$
\begin{aligned}
\left(1 \times\left. f\right|_{f^{-1}\left(x_{0}\right)}\right)_{*}\left(g \times j^{\prime}\right)^{*} M_{\mathrm{uni}} & \cong(g \times j)^{*}(f \times 1)_{*} M_{\mathrm{uni}} \\
& \cong g^{*}(1 \times j)^{*}(f \times 1)_{*} M_{\mathrm{uni}} \cong g^{*} \mathcal{V} .
\end{aligned}
$$

We now define $\phi_{\text {can }}$ to be the composition of (15) and (16).

To show that $\mathrm{G} \rightarrow$ Pres $^{\sharp} X^{+}$is an isomorphism, we construct the inverse natural transformation. Thus, suppose that $I \rightarrow\left(f_{T}\right)_{*} M$ is a family of presentations over a given $k$-scheme $T$. By definition, the cokernel $\left(f_{T}\right)_{*} M / I$ of the presentation can be written as $\left(j_{T}\right)_{*} \mathcal{W}$ for some locally free sheaf $\mathcal{W}$ on $\left\{x_{0}\right\} \times T$ of rank $b\left(x_{0}\right)-1$. We now construct a surjection from $t^{*} \mathcal{V}$ to a sheaf constructed from $\mathcal{W}$.

The line bundle $M$ defines a morphism $t: T \rightarrow \operatorname{Pic}^{-1} \tilde{X}$. The pullback $(1 \times t)^{*} M_{\text {uni }}$ may not be isomorphic to $M$, but by [1, Proposition 5.6(i)] there exists a line bundle $N$ on $T$ with the property that there exists an isomorphism $(1 \times t)^{*} M_{\text {uni }} \cong M \otimes N$. (Here we are confusing $N$ with its pullback under $X \times T \rightarrow T$, and we will continue to do so for the rest of the proof.) If we fix one such isomorphism $\alpha$, then we can consider the composition

$$
\left(i_{T}\right)^{*}\left(f_{T}\right)^{*}(1 \times t)^{*} M_{\text {uni }} \stackrel{\left(i_{T}\right)_{*}\left(f_{T}\right)_{*} \alpha}{\longrightarrow}\left(i_{T}\right)^{*}\left(f_{T}\right)_{*} M \otimes N \stackrel{(q \otimes 1)^{\#}}{\longrightarrow} \mathcal{W} \otimes N,
$$

with $(q \otimes 1)^{\sharp}$ the adjoint to $q \otimes 1:\left(f_{T}\right)_{*} M \otimes N \rightarrow\left(i_{T}\right)_{*} \mathcal{W} \otimes N$. A third application of the cohomological flatness of $f$ shows that a suitable base-change homomorphism defines an isomorphism

$$
t^{*} \mathcal{V} \cong\left(i_{T}\right)^{*}\left(f_{T}\right)_{*}(1 \times t)^{*} M_{\mathrm{uni}}
$$

and the composition of this isomorphism with the homomorphism (17) is a surjection $t^{*} \mathcal{V} \rightarrow \mathcal{W} \otimes N$ with locally free quotient of rank $b\left(x_{0}\right)-1$. To show that this 
construction defines a map (Pres $\left.X^{+}\right)(T) \rightarrow \mathrm{G}(T)$, we need to show that this surjection only depends on the equivalence class of the presentation $I \rightarrow\left(f_{T}\right)_{*} M$.

Thus suppose that we are given a second $N^{\prime}$ and a second isomorphism

$$
\alpha^{\prime}:(1 \times t)^{*} M_{\text {uni }} \cong M \otimes N^{\prime} .
$$

Because $f_{*} M$ is simple [1, Lemma 5.4], $\alpha^{\prime} \circ \alpha^{-1}: M \otimes N \cong M \otimes N^{\prime}$ must be of the form $1 \otimes \beta$ for an isomorphism $\beta: N \cong N^{\prime}$. The isomorphisms

$\left(i_{T}\right)_{*} f_{T}^{*} 1 \otimes \beta:\left(i_{T}\right)_{*} f_{T}^{*} M \otimes N \cong\left(i_{T}\right)_{*} f_{T}^{*} M \otimes N^{\prime} \quad$ and $\quad \beta \otimes 1: \mathcal{W} \otimes N \cong \mathcal{W} \otimes N^{\prime}$

define an isomorphism between the quotient associated to $(N, \alpha)$ and the quotient associated to $\left(N^{\prime}, \alpha^{\prime}\right)$. This shows that the construction from the previous paragraph defines a map (Pres $\left.X^{+}\right)(T) \rightarrow \mathrm{G}(T)$. To complete the proof, we now simply observe that the maps $\left(\operatorname{Pres} X^{+}\right)(T) \rightarrow \mathrm{G}(T)$ and $\mathrm{G}(T) \rightarrow\left(\right.$ Pres $\left.X^{+}\right)(T)$ are inverse to each other.

Lemma 3.4 does not assert that Pres ${ }^{\#} X^{+}$is representable for all $X^{+}$because a universal line bundle $M_{\text {uni }}$ does not always exist. When $M_{\text {uni }}$ fails to exist, we do not prove that Pres ${ }^{\sharp} X^{+}$is representable, but in Proposition 3.8 below we prove that the associated étale sheaf is representable. Motivated by this, we make the following definition.

Definition 3.6 Let Pres ${ }^{\text {ét }} X^{+}$be the étale sheaf associated to Pres ${ }^{\sharp} X^{+}$. A $k$-scheme Pres $X^{+}$that represents Pres ${ }^{\text {ét }} X^{+}$is called the presentation scheme.

We prove that the presentation scheme exists by reducing to Lemma 3.4, and we make the reduction by extending scalars. If $k^{\prime} \supset k$ is a field extension, then $X_{k^{\prime}}^{+}$has an associated presentation functor because, as we observed in Section $2, X_{k^{\prime}}^{+}$is described by a suitable pushout construction. Comparing the two definitions of the presentation functor, we have:

Lemma 3.7 The formations of Pres ${ }^{\sharp} X^{+}$and Pres ${ }^{\text {ét }} X^{+}$commute with field extensions $k^{\prime} \supset k$.

Proposition 3.8 The presentation scheme exists and is a projective $\mathrm{Pic}^{-1} \tilde{X}$-scheme.

Remark 3.9 As with Lemma 3.4, our proof is closely modeled on work of Altman and Kleiman [2, Theorem 12]. 
Proof When $\operatorname{Pic}^{-1} \tilde{X}$ admits a universal family of line bundles, the proposition is Lemma 3.4. In general, there exists a finite separable extension $k^{\prime} \supset k$ with the property that $\widetilde{X}_{k^{\prime}} \rightarrow \operatorname{Spec}\left(k^{\prime}\right)$ admits a section, and hence $\mathrm{Pic}^{-1} \tilde{X}_{k^{\prime}}$ admits a universal family of line bundles. Thus the presentation scheme Pres $X_{k^{\prime}}^{+}$exists. By Lemma 3.7, Pres $X_{k^{\prime}}^{+}$ represents Pres ${ }^{\text {ét }} X_{k^{\prime}}^{+}$. The $k^{\prime}$-scheme Pres $X_{k^{\prime}}^{+}$thus carries natural descent data, and to complete the proof, it is enough to show that these descent data are effective.

Consider the morphism Pres $X_{k^{\prime}}^{+} \rightarrow \mathrm{Pic}^{-1} \tilde{X}_{k^{\prime}} \times \overline{\mathrm{Pic}}^{-1} X_{k^{\prime}}^{+}$that sends $i: I \rightarrow\left(f_{T}\right)_{*} M$ to the pair $(M, I)$. The descent data on Pres $X_{k^{\prime}}^{+}$extend to descent data on this morphism. Furthermore, the morphism has finite fibers by the proof of [2, Lemma 8] (or Proposition 3.16 below). Both Pres $X_{k^{\prime}}^{+}$and $\mathrm{Pic}^{-1} \tilde{X}_{k^{\prime}} \times \overline{\mathrm{Pic}}^{-1} X_{k^{\prime}}^{+}$are $k^{\prime}$-proper, so Pres $X_{k^{\prime}}^{+} \rightarrow \mathrm{Pic}^{-1} \tilde{X}_{k^{\prime}} \times \overline{\mathrm{Pic}}^{-1} X_{k^{\prime}}^{+}$must be finite. Descent data for finite morphisms are always effective, so we can conclude that the morphism and hence Pres $X_{k^{\prime}}^{+}$descend to $k$.

The presentation scheme admits the following two morphisms.

Definition 3.10 The morphism $p_{1}$ : Pres $X^{+} \rightarrow \mathrm{Pic}^{-1} \tilde{X}$ is defined by the rule that sends a family of presentations $i: I \rightarrow f_{*} M$ to the line bundle $M$. The morphism $p_{2}$ : Pres $X^{+} \rightarrow \bar{P}^{-1} X^{+}$is defined by the rule that sends $i: I \rightarrow f_{*} M$ to $I$.

Certainly the rule sending $i: I \rightarrow f_{*} M$ to $I$ defines a morphism from Pres $X^{+}$to the moduli space of all rank-1, torsion-free sheaves, but we should explain why the rule defines a morphism into the closure $\overline{\mathrm{Pic}}^{-1} X^{+}$of the line bundle locus. The presentation scheme Pres $X^{+}$is geometrically irreducible because, by Lemma 3.4, $p_{1}$ realizes Pres $X^{+}$as a projective bundle over $\operatorname{Pic}^{-1} \tilde{X}$. We can conclude that the image of $p_{2}$ is geometrically irreducible, and the image also contains the line bundle locus because every line bundle $M$ admits a presentation; the presentation $i_{\text {can }}$. We can conclude that the image of Pres $X^{+}$is $\overline{\mathrm{P}}^{-1} X^{+}$, and in particular $p_{2}$ maps to $\overline{\mathrm{Pic}}^{-1} X^{+}$.

Next we construct some presentations.

Definition 3.11 Let $T$ be a $k$-scheme. Given $\bar{y}: T \rightarrow f^{-1}\left(x_{0}\right)$, the image of

$$
\bar{y} \times 1: T \rightarrow \tilde{X}_{T}
$$

is a Cartier divisor that we denote by $y \subset \tilde{X}_{T}$. Suppose that we are also given a line bundle $N$ on $\tilde{X}_{T}$. Then set

$$
M:=N \otimes \mathcal{O}_{\tilde{X} T}\left(f^{-1}\left(x_{0}\right)_{T}-y\right) .
$$

The divisor $f^{-1}\left(x_{0}\right)_{T}-y$ is effective, so there is a natural inclusion $i:\left(f_{T}\right)_{*} N \rightarrow$ $\left(f_{T}\right)_{*} M$. We define $(M, i)$ to be the presentation associated to $(N, \bar{y})$. 
To see that $(M, i)$ is a family of presentations, observe that on $\tilde{X}_{T}$ we have the exact sequence

$$
\left.0 \rightarrow N \rightarrow M \rightarrow M\right|_{f^{-1}\left(x_{0}\right)_{T}-y} \rightarrow 0 .
$$

Here $N \rightarrow M$ is the natural inclusion and $\left.M \rightarrow M\right|_{f^{-1}\left(x_{0}\right)_{T-y}}$ is the natural restriction. The direct image of $N \rightarrow M$ under $f_{T}$ is $i$, and so the cokernel of $i$ is

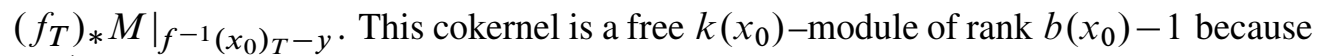
$f^{-1}\left(x_{0}\right)_{T}-y$ is a finite flat $k$-scheme of degree $b\left(x_{0}\right)-1$.

Because the associated presentation is a presentation, if $N$ is a line bundle on $\tilde{X}$ and $f^{-1}\left(x_{0}\right)$ admits a $k$-valued point, then $f_{*}(N)$ admits a presentation: the presentation associated to $(N, \bar{y})$. We now classify all the sheaves that admit a presentation.

Lemma 3.12 Assume $k=\bar{k}$. If $g: \bar{Y} \rightarrow X^{+}$is a proper birational morphism out of a curve $\bar{Y}$ with at most one singularity, then $g_{*} N$ admits a presentation for every line bundle $N$ on $\bar{Y}$.

Proof Given $\bar{Y}$, factor the normalization map $f: \tilde{X} \rightarrow X^{+}$as

$$
\tilde{X} \stackrel{h}{\longrightarrow} \bar{Y} \stackrel{g}{\longrightarrow} X^{+} .
$$

By the discussion at the end of Section 2, the curve $\bar{Y}$ can be constructed as the pushout of $\tilde{X}$ and some subset $\partial Y$ as in the diagram (11). Label the fiber $g^{-1}\left(x_{0}\right)=$ $\left\{y_{0}, y_{1}, \ldots, y_{n}\right\}$ so that the points $y_{1}, \ldots, y_{n}$ are not singularities.

On $\bar{Y}$, we have the homomorphism $i_{\text {can }}: N \rightarrow g_{*} g^{*} N$ that is adjoint to the identity. This homomorphism is injective with cokernel equal to a $k\left(y_{0}\right)$-module of rank $b\left(y_{0}\right)-1$. To see this, observe that $i_{\text {can }}$ is certainly an isomorphism away from $y_{0}$. Thus, the kernel of $i_{\text {can }}$ is supported on a proper subset of $\bar{Y}$, but this is only possible if the kernel is zero, as $N$ is torsion-free, showing injectivity. The cokernel is supported on $y_{0}$, so to compute it we can pass to an open neighborhood of $y_{0}$ and hence assume $N$ is trivial. When $N$ is trivial, the claim follows from the existence of the exact sequence (10). We now use $i_{\text {can }}$ as follows.

Because the points $y_{1}, \ldots, y_{n}$ are not singularities, the line bundle $\mathcal{O}_{\bar{Y}}\left(y_{1}+\cdots+y_{n}\right)$ is well defined, and we set $j: N \rightarrow N \otimes \mathcal{O}_{\bar{Y}}\left(y_{1}+\cdots+y_{n}\right)$ equal to the natural inclusion and $M$ equal to $h^{*}\left(N \otimes \mathcal{O}_{\bar{Y}}\left(y_{1}+\cdots+y_{n}\right)\right)$. The homomorphism

$$
f_{*} h^{*}(j) \circ g_{*}\left(i_{\text {can }}\right): g_{*} N \rightarrow f_{*} M
$$

is a presentation of $g_{*} N$. To verify this, we need to show that the cokernel is a $k\left(x_{0}\right)$-module of rank $b\left(x_{0}\right)-1$. 
We compute the cokernel using

$$
h_{*} h^{*}(j) \circ i_{\text {can }}: N \rightarrow h_{*} M .
$$

The direct image of this homomorphism under $g_{*}$ is (18).

Temporarily set $Q$ equal to the cokernel of (19). The module $Q$ must be supported on $\left\{y_{0}, \ldots, y_{n}\right\}$ because (19) equals $i_{\text {can }}$ away from $y_{1}, \ldots, y_{n}$. For the same reason, the localization $Q_{y_{0}}$ is a $k\left(y_{0}\right)$-module of rank $b\left(y_{0}\right)-1$. Away from $y_{0}$, the homomorphism (19) coincides with $j$, so the stalk of $Q$ at $y_{i}$ for $i=1, \ldots, n$ is a rank-1 module over $k\left(y_{i}\right)$. Since $g_{*} Q$ is the cokernel of the homomorphism (18), we can conclude that this cokernel is a $k\left(x_{0}\right)$-module of rank

$$
b\left(y_{0}\right)-1+1+\cdots+1=b\left(y_{0}\right)-1+n=b\left(x_{0}\right)-1 .
$$

Next we show that the only sheaves that admit a presentation are the sheaves appearing in the previous lemma.

Lemma 3.13 Assume $k=\bar{k}$. If $I$ is a rank-1, torsion-free sheaf that admits a presentation, then there exists a proper birational morphism $g: \bar{Y} \rightarrow X^{+}$out of a curve $\bar{Y}$ with at most one singularity and a line bundle $N$ on $\bar{Y}$ such that $I=g_{*} N$.

Proof Let $I$ be given. We first show that there exists $g: \bar{Y} \rightarrow X^{+}$and $N_{0}$ such that the stalks $I_{x_{0}}$ and $\left(g_{*} N_{0}\right)_{x_{0}}$ are isomorphic. We then modify $N_{0}$ to produce a suitable $N$.

We construct $\bar{Y}$ and $N_{0}$ as follows. Fix a presentation $i: I \rightarrow f_{*} M$ of $I$ with quotient $q: f_{*} M \rightarrow Q$ and consider the induced map $q_{x_{0}}: M_{f^{-1}\left(x_{0}\right)}=\left(f_{*} M\right)_{x_{0}} \rightarrow Q_{x_{0}}$ on stalks. By definition, $i_{x_{0}}\left(I_{x_{0}}\right)$ is the kernel of $q_{x_{0}}$, but we can also compute this kernel directly. The kernel must contain the product $\mathfrak{p}_{x_{0}} \cdot M_{f^{-1}\left(x_{0}\right)}$ with the maximal ideal $\mathfrak{p}_{x_{0}} \subset \mathcal{O}_{X+, x_{0}}$ because $Q$ is a $k\left(x_{0}\right)=\mathcal{O}_{X, x_{0}} / \mathfrak{p}_{x_{0}}$-module. The induced homomorphism

$$
\bar{q}: k\left(x_{0}\right) \otimes M_{f^{-1}\left(x_{0}\right)}=\bigoplus_{f(\widetilde{x})=x_{0}}(k(\tilde{x}) \otimes M) \rightarrow k\left(x_{0}\right) \otimes Q .
$$

on fibers is a surjection from a rank- $b\left(x_{0}\right)$ vector space to a rank- $\left(b\left(x_{0}\right)-1\right)$ vector space, so $\bar{q}$ has a rank-1 kernel. Pick $s_{0} \in M_{f^{-1}\left(x_{0}\right)}$ mapping to a generator of this kernel. The element $s_{0}$ itself lies in $i_{x_{0}}\left(I_{x_{0}}\right)$, and $i_{x_{0}}\left(I_{x_{0}}\right)$ is generated by $s_{0}$ together with $\mathfrak{p}_{x_{0}} \cdot M_{f^{-1}\left(x_{0}\right)}$. By using the prime avoidance lemma to modify an arbitrary $s_{0}$, we can assume that $s_{0}$ satisfies $s_{0} \notin \mathfrak{p}_{\tilde{x}}^{2} \cdot M_{\tilde{x}}$ for all $\tilde{x} \in f^{-1}\left(x_{0}\right)$.

We now use $s_{0}$ to construct $\bar{Y}$ and $N_{0}$. Define $\partial Y$ be the scheme that is the union of copies of $\operatorname{Spec}(k)$ labeled by the points $\tilde{x} \in f^{-1}\left(x_{0}\right)$ with $s_{0}(\tilde{x})=0$ plus one 
additional copy of $\operatorname{Spec}(k)$. There is a natural morphism $\partial X \rightarrow \partial Y$ that sends a $\tilde{x}$ satisfying $s_{0}(\tilde{x})=0$ to the point labeled by $\tilde{x}$ and sends every $\tilde{x}$ with $s_{0}(\tilde{x}) \neq 0$ to the additional $\operatorname{Spec}(k)$. With this $\partial X \rightarrow \partial Y$, we define $\bar{Y}$ by the diagram (11). Let

$$
f=\tilde{X} \stackrel{h}{\longrightarrow} \bar{Y} \stackrel{g}{\longrightarrow} X^{+}
$$

be the natural factorization. We claim that an isomorphism

$$
\phi_{x_{0}}:\left(g_{*} \mathcal{O}_{\bar{Y}}\right)_{x_{0}}=\mathcal{O}_{\bar{Y}, g^{-1}\left(x_{0}\right)} \cong i_{x_{0}}\left(I_{x_{0}}\right)
$$

is given by $\phi_{x_{0}}(r)=r \cdot s_{0}$. This rule defines a homomorphism $\phi_{x_{0}}: \mathcal{O}_{\bar{Y}, g^{-1}\left(x_{0}\right)} \rightarrow$ $M_{f^{-1}\left(x_{0}\right)}$ that is injective, as it is a nonzero homomorphism out of a rank-1, torsion-free module. Now let us show that the image is contained in $i_{x_{0}}\left(I_{x_{0}}\right)$.

The element $\phi_{x_{0}}(1)=s_{0}$ certainly lies in $i_{x_{0}}\left(I_{x_{0}}\right)$, but we cannot immediately conclude that $\phi_{x_{0}}(r) \in i_{x_{0}}\left(I_{x_{0}}\right)$ for all $r$ because $I_{x_{0}}$ is not (a priori) a $\mathcal{O}_{\bar{Y}, g^{-1}\left(y_{0}\right)}$-module. However, a computation shows that

$$
\begin{aligned}
q\left(r \cdot s_{0}\right)=\sum_{f(\tilde{x})=x_{0}} \bar{q}\left(r \cdot s_{0}(\tilde{x})\right)=\sum_{f(\tilde{x})=x_{0}} \bar{q}\left(r(h(\tilde{x})) s_{0}(\tilde{x})\right) & =\sum_{\tilde{x} \in \partial Y} \bar{q}\left(r\left(y_{0}\right) s_{0}(\tilde{x})\right) \\
& =r\left(y_{0}\right) q\left(s_{0}\right)=0,
\end{aligned}
$$

ie that $\phi_{x_{0}}(r)$ lies in the kernel of $q$.

To conclude that $\phi_{x_{0}}$ is an isomorphism, we need to show surjectivity. Certainly the image of $\phi_{x_{0}}$ contains $s_{0}$, so we need to show that the image also contains $\mathfrak{p}_{x_{0}} \cdot M_{f^{-1}\left(x_{0}\right)}$. A typical generator of $\mathfrak{p}_{x_{0}} \cdot M_{f^{-1}\left(x_{0}\right)}$ is $r \cdot s$ with $r \in \mathfrak{p}_{x_{0}}$ and $s \in M_{f^{-1}\left(x_{0}\right)}$. Since $M_{f^{-1}\left(x_{0}\right)}$ is generically free of rank 1 , we can certainly write $r \cdot s=r_{0} \cdot s_{0}$ for a unique rational function $r_{0}$. We show that $r_{0} \in \mathcal{O}_{\bar{Y}, g^{-1}\left(x_{0}\right)}$ by examining the stalk at a point $\tilde{x} \in f^{-1}\left(x_{0}\right)$.

If $\tilde{x} \in \partial Y$, then $M_{\tilde{x}}$ is freely generated by $s_{0}$. Writing $s=r_{1} \cdot s_{0}$ for $r_{1} \in \mathcal{O}_{X, \tilde{x}}$, we see $r_{0}=r \cdot r_{1}$, so $r_{0} \in \mathfrak{p}_{\tilde{x}} \subset \mathcal{O}_{\tilde{X}}, \tilde{x}$. If $\tilde{x} \notin \partial Y$, then $s_{0}$ does not generate $M_{\tilde{x}}$, but the section does generate $\mathfrak{p}_{\tilde{x}} \cdot M_{\tilde{x}}$, and similar reasoning shows $r_{0} \in \mathcal{O}_{\tilde{X}}, \tilde{x}$. We can conclude that $r_{0} \in \bigcap \mathcal{O}_{\tilde{X}, \tilde{x}}=\mathcal{O}_{\tilde{X}, f^{-1}\left(x_{0}\right)}$ and $r_{0}(\tilde{x})=0$ for $\tilde{x} \in \partial Y$, or equivalently $r_{0} \in \mathcal{O}_{\bar{Y}, g^{-1}\left(x_{0}\right)}$. This completes the first part of the proof, that $I_{x_{0}}$ is isomorphic to $\left(g_{*} N_{0}\right)_{x_{0}}$ for $N_{0}=\mathcal{O}_{\bar{Y}}$.

We now modify $N_{0}$ as in [21, Lemma 3.1]. We can extend the isomorphism $\phi_{x_{0}}: I_{x_{0}} \cong$ $\left(g_{*} N_{0}\right)_{x_{0}}$ to an isomorphism $\phi_{1}$ over some open neighborhood of $x_{0}$. The complement of that neighborhood is contained in some open subset over which there exists an isomorphism $\phi_{2}$ between the restrictions of $I$ and $g_{*} N_{0}$. If we define $L$ to be the line bundle given by gluing the trivial line bundle to the trivial line bundle using $\phi_{2}^{-1} \circ \phi_{1}$, 
then the isomorphisms $\phi_{1}$ and $\phi_{2}$ define an isomorphism $L \otimes g_{*} N_{0} \cong I$. The line bundle $N=g^{*} L \otimes N_{0}$ then satisfies the conditions of the lemma.

Lemma 3.14 Assume $k=\bar{k}$. Let $N$ be a line bundle on $X^{+}, M$ a line bundle on $\tilde{X}$, and $i_{x_{0}}: N_{x_{0}} \rightarrow\left(f_{*} M\right)_{x_{0}}$ an injection whose cokernel is a $k\left(x_{0}\right)-$ module. If $X^{+} \neq \tilde{X}$, then $\operatorname{rank}_{k\left(x_{0}\right)}\left(f_{*} M\right)_{x_{0}} / i_{x_{0}}\left(N_{x_{0}}\right)=b\left(x_{0}\right)-1$.

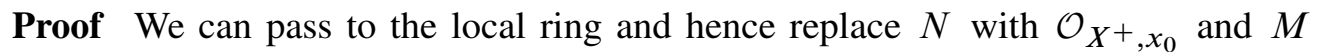
with $\mathcal{O}_{\tilde{X} f^{-1}\left(x_{0}\right)}$. The homomorphism $i$ is then given by $r \mapsto f^{*}(r) \cdot s_{0}$ for some $s_{0} \in \mathcal{O}_{\tilde{X} f^{-1}\left(x_{0}\right)}$. We claim that $s_{0}$ is a unit.

We assume $s_{0}$ is not a unit and then derive a contradiction by constructing an element of $\operatorname{Coker}(i)$ not killed by the maximal ideal $\mathfrak{p}_{x_{0}}$ of $x_{0}$. If not a unit, $s_{0}\left(\tilde{x}_{0}\right)=0$ for some $\tilde{x}_{0} \in f^{-1}\left(x_{0}\right)$. By the prime avoidance lemma, we can pick a generator $r_{0}$ of $\mathfrak{p}_{\tilde{x}_{0}}$ with the property that $r_{0}(\tilde{x}) \neq 0$ for $\tilde{x} \in f^{-1}\left(x_{0}\right), \tilde{x} \neq \tilde{x}_{0}$. The element $s:=r_{0}^{-1} \cdot s_{0}$ lies in $\mathcal{O} \tilde{X} f^{-1}\left(x_{0}\right)$, and its image in $\operatorname{Coker}(i)$ is not killed by any $t \in \mathfrak{p}_{x_{0}}$ satisfying $f^{*}(t) \notin \mathfrak{p}_{\widetilde{x}_{0}}^{2}$. Indeed, if $t$ kills $s$, then $f^{*}\left(t_{0}\right)=r_{0}^{-1} \cdot f^{*}(t)$ for some $t_{0} \in \mathcal{O}_{X^{+}, x_{0}}$. Evaluating this equation at any $\tilde{x} \neq \tilde{x}_{0}$, we see that $t_{0}\left(x_{0}\right)=0$, and by comparing orders of vanishing at $\tilde{x}_{0}$, we see that this is only possible if $f^{*}(t) \in \mathfrak{p}_{\tilde{x}_{0}}^{2}$. This shows that $\operatorname{Coker}(i)$ is not a $k\left(x_{0}\right)$-module, so we have derived the desired contradiction. We can conclude that $s_{0}$ is a unit, and so $\operatorname{Coker}(i)=\mathcal{O}_{\tilde{X} f^{-1}\left(x_{0}\right)} / f^{*}\left(\mathcal{O}_{X^{+}, x_{0}}\right)$ visibly has $k\left(x_{0}\right)$-rank $b\left(x_{0}\right)-1$.

We now classify the presentations $i: I \rightarrow f_{*} M$ with $I$ and $M$ fixed.

Lemma 3.15 Assume $k=\bar{k}$. Up to equivalence, a rank-1, torsion-free sheaf I admits at most one presentation unless $I=f_{*} N$ for $N$ a line bundle on $\tilde{X}$. When $I=f_{*} N$, there are exactly $b\left(x_{0}\right)$ inequivalent presentations: the associated presentations from Definition 3.11.

Proof By Lemma 3.13, we can assume $I=g_{*} N$ for some line bundle $N$ on a curve $\bar{Y}$ with at most one singularity. Factor $f$ as

$$
\tilde{X} \stackrel{h}{\longrightarrow} \bar{Y} \stackrel{g}{\longrightarrow} X^{+}
$$

and label the fiber $g^{-1}\left(x_{0}\right)=\left\{y_{0}, \ldots, y_{n}\right\}$ so that the points $y_{1}, \ldots, y_{n}$ are not singularities.

Suppose that $i$ is a given presentation. We can write $i$ as $i=g_{*} i^{\prime}$ for $i^{\prime}: N \rightarrow h_{*} M$ by [2, Proposition 3]. If ad( $\left.i^{\prime}\right): h^{*} N \rightarrow M$ is the adjoint to $i^{\prime}$ and $i_{\text {can }}^{\prime}: h^{*} h_{*} N \rightarrow N$ is the adjoint to the identity $h^{*} N \rightarrow h^{*} N$, then we have

$$
i^{\prime}=h_{*} \operatorname{ad}\left(i^{\prime}\right) \circ i_{\text {can }}^{\prime} .
$$


We first consider the case where $\bar{Y} \neq \tilde{X}$, the case where we need to prove that $i$ is equivalent to the presentation constructed in Lemma 3.12.

Consider the cokernel $Q^{\prime}$ of $i^{\prime}$. The direct image $g_{*} Q^{\prime}$ is the cokernel of $i$, so, since $g_{*} Q^{\prime}$ is a $k\left(x_{0}\right)$-module of rank $b\left(x_{0}\right)-1$, it follows that $Q^{\prime}$ is a module over $k\left(y_{0}\right) \oplus k\left(y_{1}\right) \oplus \cdots \oplus k\left(y_{n}\right)$ and the sum $\sum \operatorname{rank}_{k\left(y_{i}\right)} Q_{y_{i}}^{\prime}$ is $b\left(x_{0}\right)-1$. We have $\operatorname{rank}_{k\left(y_{0}\right)} Q_{y_{0}}^{\prime}=b\left(y_{0}\right)-1$ by Lemma 3.14, and, for $i \neq 0$, the $k\left(y_{i}\right)$-rank of $Q_{y_{i}}^{\prime}$ is at most 1 , as this module is a quotient of the rank-1 module $\left(h_{*} M\right)_{y_{i}}$. Combining these inequalities, we get

$$
\begin{aligned}
b\left(x_{0}\right)-1 & =\operatorname{rank}_{k\left(y_{0}\right)} Q_{y_{0}}+\cdots+\operatorname{rank}_{k\left(y_{n}\right)} Q_{y_{n}} \\
& \leq b\left(y_{0}\right)-1+1+\cdots+1=b\left(y_{0}\right)-1+n=b\left(x_{0}\right)-1,
\end{aligned}
$$

so all the inequalities must be equalities. In other words, $Q_{y_{i}}$ is a rank-1 module over $k\left(x_{i}\right)$ for all $i \neq 0$, and $Q_{y_{0}}$ has rank $b\left(y_{0}\right)-1$.

The cokernel of $\left(i_{\text {can }}^{\prime}\right)_{y_{0}}: N_{y_{0}} \rightarrow\left(h_{*} h^{*} N\right)_{y_{0}}$ is a $k\left(y_{0}\right)$-module of rank $b\left(y_{0}\right)-1$ (this was a computation in Lemma 3.12), so $h_{*}\left(\operatorname{ad}\left(i^{\prime}\right)\right)_{y_{0}}$ must be an isomorphism. Similar reasoning shows that $h_{*}\left(\operatorname{ad}\left(i^{\prime}\right)\right)_{y_{i}}$ has a rank-1 kernel. In other words,

$$
\operatorname{ad}\left(i^{\prime}\right): h^{*} N \rightarrow M
$$

has cokernel isomorphic to $k\left(\tilde{y}_{1}\right) \oplus \cdots \oplus k\left(\tilde{y}_{n}\right)$. Equivalently, ad $\left(i^{\prime}\right)$ factors as

$$
h^{*} N \rightarrow \mathcal{O}_{\tilde{X}}\left(\tilde{y}_{1}+\cdots+\tilde{y}_{n}\right) \otimes h^{*} N \rightarrow M,
$$

with $\mathcal{O}_{\tilde{X}}\left(\tilde{y}_{1}+\cdots+\tilde{y}_{n}\right) \otimes h^{*} N \rightarrow M$ an isomorphism (by degree considerations). This isomorphism defines an equivalence between the given presentation and the presentation from Lemma 3.12.

To complete the proof, we must consider the case $\bar{Y}=\tilde{X}$. Most of the argument given in the previous case remains valid except that the rank of $k\left(y_{0}\right) \otimes M_{y_{0}}$ is $b\left(y_{0}\right)=1$, not $b\left(y_{0}\right)-1=0$. Thus we can only conclude from the fact that $b\left(x_{0}\right)-1=$ $\sum \operatorname{rank}_{k\left(y_{i}\right)} Q_{y_{i}}$ that the rank of $Q_{y_{i}}$ is 1 for all but exactly one $i=i_{0}$, in which case $\operatorname{rank}_{k\left(y_{i_{0}}\right)} Q_{y_{i_{0}}}=0$. The rest of the argument shows that the given presentation $(i, M)$ is equivalent to the presentation associated to $M$ and $y_{i_{0}}$.

The following proposition summarizes the past four lemmas.

Proposition 3.16 Assume $k=\bar{k}$. Then a rank-1, torsion-free sheaf $I$ on $X^{+}$admits a presentation $i: I \rightarrow f_{*} M$ if and only there exists a curve $\bar{Y}$ with at most one singularity, a proper birational morphism $g: \bar{Y} \rightarrow X^{+}$, and a line bundle $N$ on $\bar{Y}$ such that $I=g_{*} N$. 
Furthermore, the presentation is unique unless $\bar{Y}=\tilde{X}$ is the normalization, in which case every presentation is isomorphic to the presentation associated to some $(M, \bar{y})$. In particular, there are exactly $b\left(x_{0}\right)$ different presentations of $I$.

Using this proposition, we can immediately construct rank-1, torsion-free sheaves that are not limits of line bundles. For example:

Corollary 3.17 Assume char $k>3$. Define $X^{+}$by the pushout diagram (9) with $\tilde{X}:=\mathbb{P}^{1}$ and $\partial X:=\{ \pm 1, \pm 2\}$. Define $\bar{Y}$ by the pushout diagram (11) with $\partial Y:=$ $\{ \pm 1, \pm 2\}$ and $\partial X \rightarrow \partial Y$ the morphism $\pm 1 \mapsto 1, \pm 2 \mapsto 2$. If $g: \bar{Y} \rightarrow X^{+}$is the natural morphism, then

$$
I=g_{*} \mathcal{O}_{\bar{Y}}
$$

is not the limit of line bundles.

Proof If we fix a degree- 1 line bundle $L_{0}$ on $X^{+}$, then the map Pres $X^{+} \rightarrow \overline{\operatorname{Pic}}^{0} X^{+}$ that sends $i: I \rightarrow f_{*} M$ to $I \otimes L_{0}$ is surjective, and the image does not contain $\left[g_{*} \mathcal{O}_{\bar{Y}}\right]$ by Proposition 3.16 .

We now use the result just proven to describe the structure of $\bar{P}$ ic $X^{+}$.

Definition 3.18 Define the canonical embedding into the presentation scheme

$$
\hat{\epsilon}: \operatorname{Pic}^{0} \tilde{X} \times f^{-1}\left(x_{0}\right) \rightarrow \operatorname{Pres} X^{+}
$$

by the rule that sends $(N, \bar{y}) \in\left(\operatorname{Pic}^{0} \tilde{X}\right)(T) \times f^{-1}\left(x_{0}\right)(T)$ to the associated presentation of $\left(N \otimes \mathcal{O}_{\tilde{X} T}\left(-\partial X_{T}\right), \bar{y}\right)$.

The canonical embedding into the compactified Picard scheme

$$
\epsilon: \operatorname{Pic}^{0} \tilde{X} \rightarrow \overline{\operatorname{Pic}}^{-1} X^{+}
$$

is defined by the rule $L \mapsto f_{*}\left(L \otimes \mathcal{O}_{\tilde{X} T}\left(-\partial X_{T}\right)\right)$.

The canonical embedding into the compactified Picard scheme is a closed embedding by [2, Section 5, page 19] (which deduces this property by using [2, Proposition 3] to assert that $\epsilon$ is a monomorphism), and the same argument shows that $\hat{\epsilon}$ is a closed embedding.

Proposition 3.16 allows us to describe $\overline{\mathrm{P}} \mathrm{ic}^{-1} X^{+}$up to universal homeomorphism as follows. By [15, Theorem 5.4], we may define $\bar{P}^{\natural}$ by the pushout diagram

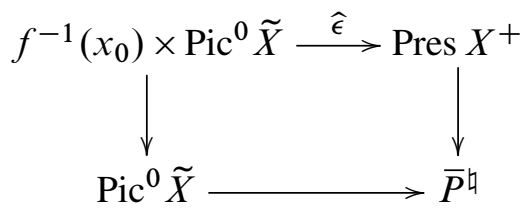


where $f^{-1}\left(x_{0}\right) \times \operatorname{Pic}^{0} \tilde{X} \rightarrow \operatorname{Pic}^{0} \tilde{X}$ is the projection morphism. Because the diagram

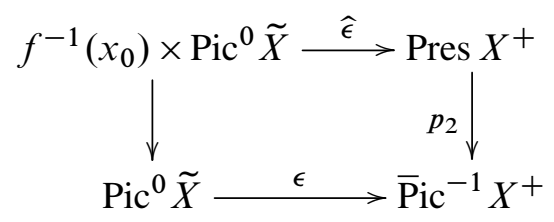

commutes, the universal property of the pushout defines a map $\bar{P}^{\natural} \rightarrow \overline{\mathrm{P}}^{-1} X^{+}$.

Theorem 3.19 The map $\bar{P}^{\natural} \rightarrow \overline{\mathrm{P}}^{-1} X^{+}$is a universal homeomorphism.

Proof The morphism $\bar{P}^{\natural} \rightarrow \overline{\mathrm{Pic}}^{-1} X^{+}$is surjective because, as we observed after the construction of Pres $X^{+}$, the morphism Pres $X^{+} \rightarrow \bar{P}^{-1} X^{+}$is surjective. Because both Pres $X^{+}$and $\overline{\mathrm{Pic}}^{-1} X^{+}$are $k$-proper, it is enough to show that the induced map $\bar{P}^{\natural}(\bar{k}) \rightarrow\left(\overline{\mathrm{Pic}}^{-1} X^{+}\right)(\bar{k})$ on $\bar{k}$-points is injective [18, Proposition 2.4.5, page 20].

We can describe the $\bar{k}$-valued points of $\bar{P}^{\natural}$ explicitly. Applying the functor taking a scheme to the set of its $\bar{k}$-points sends (21) to a pushout diagram, as can be verified,

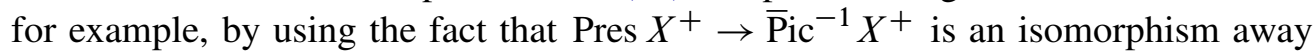
from the image of the canonical embedding and that (21) is a pullback diagram [15, Theorem 5.4]. Because the formation of the presentation scheme commutes with field extensions, $\left(\operatorname{Pres} X^{+}\right)(\bar{k})=\left(\operatorname{Pres} X_{\bar{k}}^{+}\right)(\bar{k})$, so $\bar{P}^{\natural}(\bar{k}) \rightarrow\left(\overline{\mathrm{Pic}}^{-1} X^{+}\right)(\bar{k})$ is bijective by Proposition 3.16 .

\section{The Abel map}

Here we study the Abel map of the curve $X^{+}$from Section 2. We first compare the definitions of two different Abel maps associated to a smooth curve over $k$ and show that only one of them naturally extends to $X^{+}$. Second, we lift the AltmanKleiman Abel map Ab: $X^{+} \rightarrow \bar{P}^{-1} X^{+}$to a morphism $\tilde{X} \rightarrow$ Pres $X^{+}$from the normalization to the presentation scheme. In Section 6 we will use this lifted Abel map to compute $\mathrm{H}_{1}(\mathrm{Ab})$.

The Abel map of a smooth curve $\tilde{X}$ over $k$ is defined in standard texts such as [28] to be the map $\tilde{X} \rightarrow \operatorname{Pic}^{1} \tilde{X}$ that sends a point $x$ to the line bundle $\mathcal{O}_{\tilde{X}}(x)$, which is the dual $\mathcal{O}_{\tilde{X}}(x):=\underline{\operatorname{Hom}}\left(I_{x}, \mathcal{O}_{\tilde{X}}\right)$ of the ideal $I_{x}$ of $x$. We denote this morphism by $\mathrm{Ab}^{\vee}: \tilde{X} \rightarrow \operatorname{Pic}^{1} \tilde{X}$ to distinguish it from the morphism $\mathrm{Ab}: \tilde{X} \rightarrow \mathrm{Pic}^{-1} \tilde{X}$ studied by Altman and Kleiman in [1]. There the authors define the Abel map Ab: $\tilde{X} \rightarrow \operatorname{Pic}^{-1} \tilde{X}$ to be the map that sends a point $x$ to its ideal sheaf $I_{x}$. 
Altman and Kleiman prove that this second definition extends to singular curves: given a singular curve $\bar{X}$, the rule $x \mapsto I_{x}$ defines a morphism Ab: $\bar{X} \rightarrow \overline{\mathrm{P}} \mathrm{ic}^{-1} \bar{X}$ that is a closed embedding provided the genus of $\bar{X}$ is at least 1 [1, Theorem 8.8]. We call this map the Altman-Kleiman Abel map of $\bar{X}$.

The first definition, the definition of $\mathrm{Ab}^{\vee}$, does not always extend to singular curves. This issue is slightly subtle. To show that the rule

$$
x \mapsto \underline{\operatorname{Hom}}\left(I_{x}, \mathcal{O}_{\bar{X}}\right)
$$

defines a morphism $\bar{X} \rightarrow \overline{\operatorname{Pic}^{1}} \bar{X}$, one must show that a flat deformation of $x$ induces a flat deformation of $\underline{\operatorname{Hom}}\left(I_{x}, \mathcal{O}_{\bar{X}}\right)$. That is, given a $k$-morphism $x(t): T \rightarrow \bar{X}_{T}$, one must show that the duals $\underline{\operatorname{Hom}}\left(I_{x\left(t_{0}\right)}, \mathcal{O}_{\bar{X}}\right)$ of the ideals of the fibers $x\left(t_{0}\right)$ of $x(t)$ fit together to form the fibers of an $\mathcal{O}_{T}$-flat family of sheaves on $\bar{X}_{T}$.

When $x(t)$ maps into the smooth locus $X:=\bar{X}^{\text {sm }}$, essentially the same construction as in the smooth case produces a suitable family of sheaves, and so (22) defines a morphism $\mathrm{Ab}^{\vee}: X \rightarrow \mathrm{Pic}^{1} \bar{X}$ from the smooth locus to the Picard scheme. We call this morphism the classical Abel map. When $\bar{X}$ is Gorenstein, the classical Abel map extends to a morphism defined on all of $\bar{X}$ because a construction using cohomology and base change produces a suitable family for an arbitrary $x(t)$. (For the construction, see [22, Definition 5.0.7]. The argument is a modification of [12, (2.2)].)

The one-point compactification $X^{+}$is Gorenstein, however, only when $b\left(x_{0}\right)=2$. When $b\left(x_{0}\right) \geq 3$, not only does the construction just reviewed fail to produce a morphism $X^{+} \rightarrow \bar{P}^{1}{ }^{1} X^{+}$extending the classical Abel map $\mathrm{Ab}^{\vee}$, but it may be impossible to extend the classical Abel map to a morphism out of $X^{+}$by any construction. We demonstrate this with an example.

Example 4.1 The classical Abel map $\mathrm{Ab}^{\vee}: X \rightarrow \mathrm{Pic}^{1} X^{+}$can fail to extend to a morphism $\mathrm{Ab}^{\vee}: X^{+} \rightarrow \overline{\mathrm{P}}^{1}{ }^{1} X^{+}$.

Proof of example Let $k$ be a field of characteristic not 2. Define $X^{+}$by the pushout diagram (9) with $\tilde{X}:=\mathbb{P}^{1}$ and $\partial X:=\{0,1,-1\}$, so $X^{+}$is a rational curve with a unique singularity $x_{0}$ that is an ordinary 3 -fold point. We will show that the classical Abel map $\mathrm{Ab}^{\vee}$ of $X^{+}$is undefined at $x_{0}$.

We argue as follows. The composition $\mathrm{Ab}^{\vee} \circ f: \tilde{X}-f^{-1}\left(x_{0}\right) \rightarrow \overline{\text { Pic }} X^{+}$extends to a regular map out of $\tilde{X}=\mathbb{P}^{1}$ by the valuative criterion, and we show directly that the 
points $\mathrm{Ab}^{\vee} \circ f(0), \mathrm{Ab}^{\vee} \circ f(1)$, and $\mathrm{Ab}^{\vee} \circ f(-1)$ are distinct. More precisely,

$$
\begin{aligned}
\mathrm{Ab}^{\vee} \circ f(0) & =\left[\left(g_{0}\right)_{*} \mathcal{O}_{\bar{Y}_{0}}\right], \\
\mathrm{Ab}^{\vee} \circ f(1) & =\left[\left(g_{1}\right)_{*} \mathcal{O}_{\bar{Y}_{1}}\right], \\
\mathrm{Ab}^{\vee} \circ f(-1) & =\left[\left(g_{-1}\right)_{*} \mathcal{O}_{\bar{Y}_{-1}}\right],
\end{aligned}
$$

where $g_{0}: \bar{Y}_{0} \rightarrow X^{+}, g_{-1}: \bar{Y}_{-1} \rightarrow X^{+}$, and $g_{1}: \bar{Y}_{1} \rightarrow X^{+}$are the three nodal curves lying between $X^{+}$and $\tilde{X}$. So, $\bar{Y}_{0}$ is the pushout of $\tilde{X}=\mathbb{P}^{1}$ and $\{1,-1\}$ over $\operatorname{Spec}(k)$ etc.

We verify (23) for the point 0 and leave the remaining cases (which involve only notational changes to the argument given) to the interested reader. To verify the equation, we use the dualizing sheaf $\omega$. Recall that the rule $I \mapsto \underline{\operatorname{Hom}}(I, \omega)$ defines an involution on the set of rank-1, torsion-free sheaves and the formation of $\underline{\operatorname{Hom}}(I, \omega)$ commutes with families [12, (2.2)]. We verify (23) by constructing a family of sheaves such that the dual family defines $\mathrm{Ab}^{\vee} \circ f$.

The dualizing module $\omega$ can be described as the module of Rosenlicht differentials, ie rational differentials on $\tilde{X}$ with at worst simple poles at $0,-1$ and 1 and satisfying the condition that the residues at these points sum to zero. Because a section of $\omega$ has at worst a simple pole at 0 , the rule $\phi(p(t) / q(t) d t)=a p(a) / q(a)$ defines a homomorphism

$$
\phi: \omega \otimes k[a]\left[1 /\left(a^{2}-1\right)\right] \rightarrow k[a]\left[1 /\left(a^{2}-1\right)\right] .
$$

Let $I_{a}$ be the kernel. Note that, away from $a=0$, this kernel consists of those differentials in $\omega$ which vanish at $t=a$. At $a=0$, this kernel consists of such differentials whose residue at 0 is 0 . In other words, for $\alpha \neq 0,-1,1$, the fiber $I_{\alpha}$ of $I_{a}$ over $\operatorname{Spec}(k[a] /(a-\alpha)) \rightarrow \operatorname{Spec}\left(k[a]\left[1 /\left(a^{2}-1\right)\right]\right)$ is $\omega(-f(a))$, and $I_{0}$ is the sheaf of rational differentials on $\tilde{X}$ with at worst simple poles at -1 and 1 and satisfying the condition that the residues sum to zero. By Rosenlicht's definition (or description) of $\left(g_{0}\right)_{*} \omega_{Y_{0}}$, we see that $I_{0}=\left(g_{0}\right)_{*} \omega_{Y_{0}}$.

The kernel $\operatorname{Ker} \phi=I_{a}$ is $k[a]\left[1 /\left(a^{2}-1\right)\right]$-flat because $\phi$ is surjective.

Furthermore, the $\omega$-dual $\underline{\operatorname{Hom}}\left(I_{a}, \omega\right)$ defines $\mathrm{Ab}^{\vee} \circ f$. Indeed, $\underline{\operatorname{Hom}}\left(I_{a}, \omega\right)$ is flat and its formation commutes with passing to fibers by [12, (2.2)]. Since $I_{a}=\omega(-f(a))$ for $\alpha \neq 0,-1,1$, we deduce

$$
\mathrm{Ab}^{\vee} \circ f(0)=\left[\underline{\operatorname{Hom}}\left(I_{0}, \omega\right)\right] .
$$

By the above, $I_{0}=\left(g_{0}\right)_{*} \omega_{Y_{0}}$. The morphism

$$
\left(g_{0}\right)_{*} \mathcal{O}_{Y_{0}} \rightarrow \underline{\operatorname{Hom}}\left(g_{*} \omega_{Y_{0}}, \omega\right)
$$


defined by sending a rational function to multiplication by that function defines an isomorphism, showing that $\mathrm{Ab}^{\vee} \circ f(0)=\left(g_{0}\right)_{*} \mathcal{O}_{Y_{0}}$, as claimed.

While the classical Abel map $\mathrm{Ab}^{\vee}$ may not be defined on all of $X^{+}$, the AltmanKleiman Abel map $\mathrm{Ab}$ is not only defined on all of $X^{+}$, but it admits a natural lift to a morphism Pres(Ab): $\tilde{X} \rightarrow \operatorname{Pres} X$. Given $x=f(\tilde{x})$ with $\tilde{x} \in X \subset \tilde{X}$, the ideal sheaf $I_{x}$ of $x$ in $X^{+}$admits the presentation $i_{\text {can }}: I_{x} \rightarrow f_{*} I_{\tilde{x}}=f_{*} f^{*} I_{x}$ defined in (12). The resulting morphism $X \rightarrow \operatorname{Pres} X^{+}$extends to the morphism Pres(Ab): $\tilde{X} \rightarrow \operatorname{Pres} X^{+}$ that we now define.

Definition 4.2 Let $\Gamma \subset X^{+} \times \tilde{X}$ be the transpose of the graph of $f$ and $\tilde{\Delta} \subset \tilde{X} \times \tilde{X}$ to be the diagonal. Define $I_{\tilde{x}(t)}$ to be the ideal of $\widetilde{\Delta}$ and $J_{t}$ the ideal of $\Gamma$.

Lemma 4.3 The natural inclusion $i: J_{t} \rightarrow(f \times 1)_{*} I_{\widetilde{x}(t)}$ is a family of presentations over $\tilde{X}$.

Proof We prove that $i$ is family of presentations by showing that $\operatorname{Coker}(i)$ is isomorphic to $(\bigoplus k(\tilde{x})) / k\left(x_{0}\right) \otimes \mathcal{O}_{\tilde{X}}$. This last module is the cokernel of the inclusion $\mathcal{O}_{X^{+} \times \tilde{X}} \rightarrow \mathcal{O}_{\tilde{X}} \times \tilde{X}$, as the formation of the pushout $X^{+}$commutes with the flat base change $X^{+} \times \tilde{X} \rightarrow X^{+}$. All of the modules in question fit into the following commutative diagram with exact rows and columns:

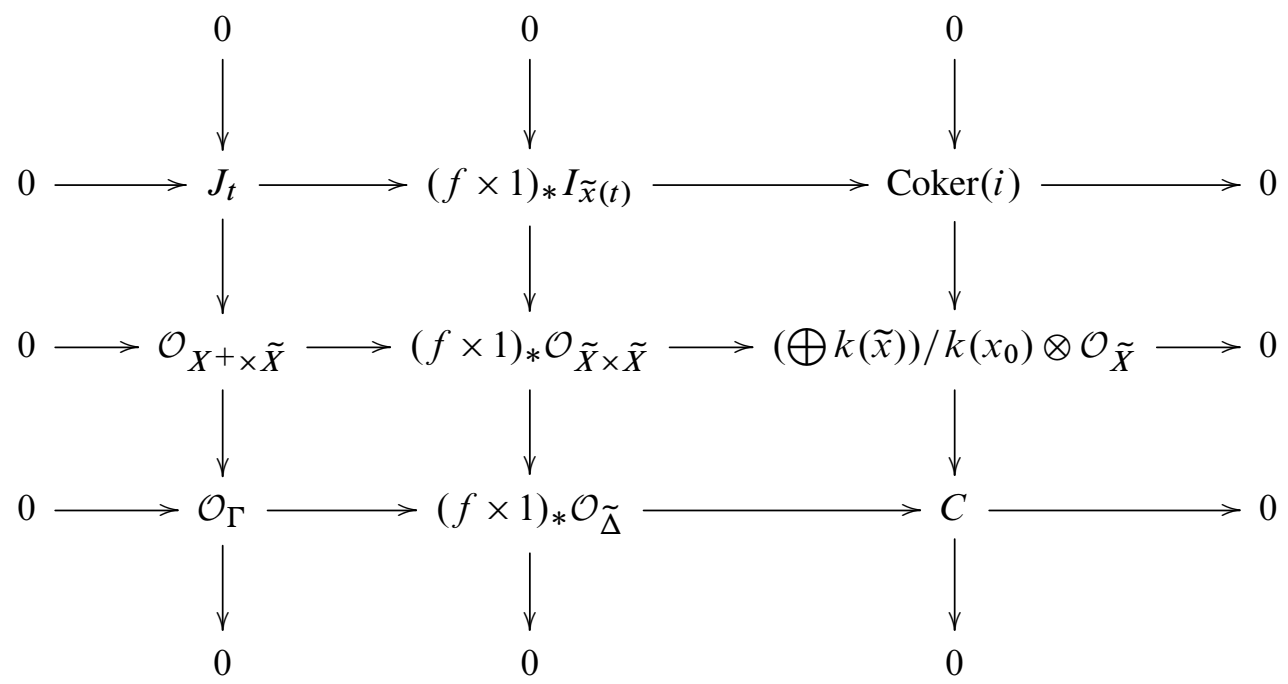

The existence of the diagram follows by, for example, applying the snake lemma to the first two columns. In the diagram, $\mathcal{O}_{\Gamma}$ and $\mathcal{O}_{\tilde{\Delta}}$ are structure sheaves, and $C$ is the cokernel of $\operatorname{Coker}(i) \rightarrow(\bigoplus k(\tilde{x})) / k\left(x_{0}\right)$ or equivalently of $\mathcal{O}_{\Gamma} \rightarrow(f \times 1)_{*} \mathcal{O}_{\tilde{\Delta}}$. 
Now $\Gamma$ and $\widetilde{\Delta}$ are both images of $\tilde{X}$ under a closed embedding, and under the associated identifications $\Gamma=\tilde{X}, \widetilde{\Delta}=\tilde{X}$, the morphism $f \times 1: \widetilde{\Delta} \rightarrow \Gamma$ becomes identified with the identity. In particular, the natural morphism $\mathcal{O}_{\Gamma} \rightarrow(f \times 1)_{*} \mathcal{O}_{\widetilde{\Delta}}$ is an isomorphism and so $C=0$. We can conclude that the natural morphism

$$
\operatorname{Coker}(i) \rightarrow(\bigoplus k(\tilde{x})) / k\left(x_{0}\right) \otimes \mathcal{O}_{\tilde{X}}
$$

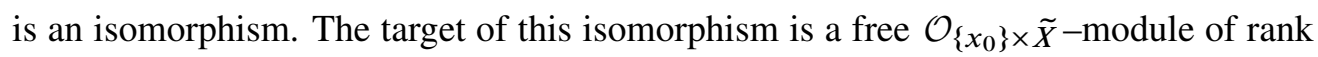
$b\left(x_{0}\right)-1$, so we can conclude that $i$ is a family of presentations.

Definition 4.4 We define the lifted Abel map Pres(Ab): $\tilde{X} \rightarrow \operatorname{Pres} X^{+}$to be the morphism defined by the family of presentations from Lemma 4.3.

By construction, the lifted Abel map has the property that the composition $p_{1} \circ \operatorname{Pres}(\mathrm{Ab})$ is the Altman-Kleiman Abel map $\widetilde{\mathrm{A}} b$ of $\tilde{X}$, and the composition $p_{2} \circ \operatorname{Pres}(\mathrm{Ab})$ is the composition Abof of Altman-Kleiman Abel map of $X^{+}$with the normalization map.

\section{Cohomology of $X$ via Pic}

Let $X$ be a smooth curve over $k$, contained as an open subset of $\tilde{X}$, which is smooth and proper. Assume that $\tilde{X}$ has genus greater than 0, so its Abel map is non-trivial. We show that $\mathrm{H}^{1}\left(X_{\bar{k}}, R(1)\right)$ with its $\operatorname{Gal}(\bar{k} / k)$-module structure is obtained from the fundamental groupoid of $\operatorname{Pic}^{-1} \tilde{X}$. When $\tilde{X}$ has genus 0 , a degenerate form of this result holds, given in Remark 5.3.

For $R=\mathbb{Z} / \ell^{m}$ (respectively $\mathbb{Z}_{\ell}$ ), let $\mathcal{U}_{R}$ denote the functor taking a finitely generated $R$-module to the underlying (topological) groupoid. Under suitable finiteness hypotheses on the category of groupoids, say connected groupoids with finitely many objects and finitely (topologically) generated morphism spaces, $\mathcal{U}_{R}$ has a left adjoint, denoted $\mathcal{F}_{R}$.

For such a groupoid $\pi$, with objects $D$ and source and target maps $s, t: \pi \rightarrow D$, respectively, there is a canonical functorial exact sequence

$$
0 \longrightarrow \mathcal{F}_{R} \pi_{1} \longrightarrow \mathcal{F}_{R} \pi \stackrel{t-s}{\longrightarrow} \bigoplus_{D} R \stackrel{\oplus_{D} \text { id }}{\longrightarrow} R \longrightarrow 0,
$$

where $\pi_{1}$ is the sub-groupoid spanned by any single object of $D$. To see that (24) is canonical, note that the group $\mathcal{F}_{R} \pi_{1}$ is independent of the choice of object defining $\pi_{1}$ because a morphism $d_{s} \rightarrow d_{t}$ in $\pi$ determines an isomorphism $\pi_{d_{s}} \rightarrow \pi_{d_{t}}$ between the corresponding sub-groupoids, and applying $\mathcal{F}_{R}$ gives an isomorphism $\mathcal{F}_{R} \pi_{d_{s}} \rightarrow \mathcal{F}_{R} \pi_{d_{t}}$ independent of the choice of morphism from $d_{s}$ to $d_{t}$. 
Let $\partial X \subsetneq \tilde{X}$ be a closed subscheme with open complement $X$. For each point $\tilde{x}$ of $\partial X_{\bar{k}}$ choose a $\bar{k}$-geometric point with image $\tilde{x}$, and let $\partial X(\bar{k})$ be the set of these chosen geometric points. We may assume that $\partial X(\bar{k})$ is stable under the $\mathrm{Gal}(\bar{k} / k)$-action.

Theorem 5.1 There is a canonical functorial isomorphism of $\mathrm{Gal}(\bar{k} / k)$-modules

$$
\mathcal{F}_{R} \pi_{1}^{\ell}\left(\mathrm{Pic}^{-1} \tilde{X}_{\bar{k}}, \mathrm{Ab}_{*} \partial X(\bar{k})\right) \cong \mathrm{H}^{1}\left(X_{\bar{k}}, R(1)\right) .
$$

Remark 5.2 When $\partial X=\varnothing$ one may take $\mathrm{Ab}_{*} \partial X(\bar{k})$ to be the set consisting of any single geometric point of $\mathrm{Pic}^{-1} \tilde{X}_{\bar{k}}$.

Remark 5.3 When $\tilde{X}$ has genus 0 , we have a canonical functorial short exact sequence of $\operatorname{Gal}(\bar{k} / k)$-modules

$$
0 \longrightarrow \mathrm{H}^{1}\left(X_{\bar{k}}, R(1)\right) \longrightarrow \bigoplus_{\partial X(\bar{k})} R \stackrel{\oplus_{\partial X(\bar{k})} \text { id }}{\longrightarrow} R \longrightarrow 0
$$

This follows from applying cohomology to the pair $\left(X_{\bar{k}}, \tilde{X}_{\bar{k}}\right)$. Compare with (24) and Theorem 5.1.

Proof Multiplication by $\ell^{n}$

$$
m_{\ell^{n}}: \operatorname{Pic}^{0} \tilde{X}_{\bar{k}} \rightarrow \operatorname{Pic}^{0} \tilde{X}_{\bar{k}}
$$

is a finite étale map. For a geometric point $a$ of $\operatorname{Pic}^{0} \tilde{X}_{\bar{k}}$, let $\phi_{a}\left[m_{\ell}\right]$, abbreviated $\phi_{a}\left[\ell^{n}\right]$, denote the fiber above $a$.

For every $n$ and $\tilde{x}_{s}, \tilde{x}_{t}$ in $\partial X(\bar{k})$, there is a canonical, functorial map

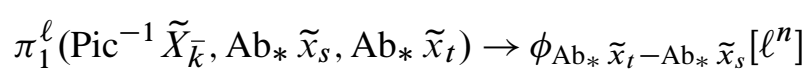

constructed as follows.

Addition by a $\bar{k}$-point $a$ of $\operatorname{Pic}^{1} \tilde{X}_{\bar{k}}$ defines $+_{a}: \operatorname{Pic}^{-1} \tilde{X}_{\bar{k}} \rightarrow \operatorname{Pic}^{0} \tilde{X}_{\bar{k}}$. Pulling back $m_{\ell^{n}}$ by $+a$ produces a finite étale map to $\operatorname{Pic}^{-1} \tilde{X}_{\bar{k}}$, whence a map

$$
\pi_{1}^{\ell}\left(\mathrm{Pic}^{-1} \tilde{X}_{\bar{k}}, \mathrm{Ab}_{*} \tilde{x}_{s}, \mathrm{Ab}_{*} \tilde{x}_{t}\right) \rightarrow \operatorname{Mor}\left(\phi_{\mathrm{Ab}_{*} \tilde{x}_{s}}\left[+{ }_{a}^{*} m_{\ell^{n}}\right], \phi_{\mathrm{Ab}_{*} \tilde{x}_{t}}\left[+{ }_{a}^{*} m_{\ell^{n}}\right]\right) .
$$

Since elements of $\pi_{1}^{\ell}$ are natural transformations between fiber functors, the image is contained in the morphisms

$$
\left.\phi_{a+\mathrm{Ab} * \tilde{x}_{s}}\left[\ell^{n}\right] \cong \phi_{\mathrm{Ab}_{*} \tilde{x}_{s}}\left[+_{a}^{*} m_{\ell^{n}}\right] \rightarrow \phi_{\mathrm{Ab} *} \tilde{x}_{t}\left[+{ }_{a}^{*} m_{\ell^{n}}\right]\right) \cong \phi_{a+\mathrm{Ab}_{*} \tilde{x}_{t}}\left[\ell^{n}\right]
$$

given by addition by an element of $\phi_{\mathrm{Ab} *} \tilde{x}_{t}-\mathrm{Ab}_{*} \tilde{x}_{s}\left[\ell^{n}\right]$. Sending the morphism to this element of $\phi_{\mathrm{Ab}_{*} \tilde{x}_{t}-\mathrm{Ab}_{*} \tilde{x}_{s}}\left[\ell^{n}\right]$ defines (25), which is independent of the choice of $a$. 
By the moduli definition of the Picard functor, the $\bar{k}$-points of Pic $\tilde{X}_{\bar{k}}$ determine invertible sheaves on $\tilde{X}_{\bar{k}}$. For the image $\tilde{x}$ in $\tilde{X}_{\bar{k}}$ of any element of $\partial X(\bar{k})$, the restriction of $\mathcal{O} \tilde{X}_{\bar{k}}(\tilde{x})$ to $X_{\bar{k}}$ has a canonical trivialization coming from the inclusion of the ideal sheaf $I_{\tilde{x}}=\mathcal{O}_{\tilde{X}_{\bar{k}}}(-\tilde{x})$ into $\mathcal{O}_{\tilde{X}_{\bar{k}}}$ which becomes an isomorphism after pullback to $X_{\bar{k}}$. Thus elements of $\phi_{\mathrm{Ab} *} \tilde{x}_{t}-\mathrm{Ab}_{*} \tilde{x}_{s}\left[\ell^{n}\right]$ determine an invertible sheaf $L$ on $X_{\bar{k}}$ equipped with a trivialization of $\otimes{ }^{\ell^{n}} L$.

The data of an invertible sheaf $L$ on $X_{\bar{k}}$ equipped with a trivialization of $\otimes^{\ell^{n}} L$ produces a canonical element of $\mathrm{H}^{1}\left(X_{\bar{k}}, \mathbb{Z} / \ell^{n}(1)\right)$, as follows: there is a map $L \rightarrow$ $\otimes^{\ell^{n}} L$ taking a section $a$ of $L$ over a Zariski-open $U$ to $\otimes^{\ell^{n}} a$. The coherent sheaves $L$ and $\otimes^{\ell^{n}} L$ determine $\mathbb{G}_{m}$-torsors, which we view as sheaves of sets in the étale topology equipped with an action of $\mathbb{G}_{m}$. Under this identification $a \mapsto \bigotimes^{\ell^{n}} a$ respects the actions of $\mathbb{G}_{m}$ in the sense that, for $b \in \mathbb{G}_{m}$,

$$
a \cdot b \mapsto \bigotimes^{\ell^{n}} a \cdot b^{\ell^{n}}
$$

There is an element 1 in the set of sections over any étale open of the $\mathbb{G}_{m}$-torsor $\otimes^{\ell^{n}} L$ determined by the chosen trivialization of $\bigotimes^{\ell^{n}} L$. The sections $s$ of the $\mathbb{G}_{m}$-torsor $L$ such that $\otimes^{\ell^{n}} s$ equals 1 determine a $\mu_{\ell^{n}}$-torsor, whence a canonical element of $\mathrm{H}^{1}\left(X_{\bar{k}}, \mathbb{Z} / \ell^{n}(1)\right)$.

Thus there is a canonical, functorial map

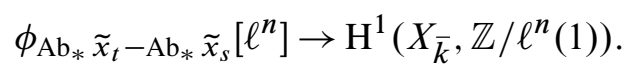

Composing (25) and (26) determines a $\operatorname{Gal}(\bar{k} / k)$-equivariant map

$$
\pi_{1}^{\ell}\left(\mathrm{Pic}^{-1} \tilde{X}_{\bar{k}}, \mathrm{Ab}_{*} \partial X(\bar{k})\right) \rightarrow \mathrm{H}^{1}\left(X_{\bar{k}}, R(1)\right),
$$

whence a map

$$
\theta: \mathcal{F}_{R} \pi_{1}^{\ell}\left(\mathrm{Pic}^{-1} \tilde{X}_{\bar{k}}, \mathrm{Ab}_{*} \partial X(\bar{k})\right) \rightarrow \mathrm{H}^{1}\left(X_{\bar{k}}, R(1)\right),
$$

which we will show to be an isomorphism.

Applying $\mathrm{H}^{*}(-, R(1))$ to the pair $\left(X_{\bar{k}}, \tilde{X}_{\bar{k}}\right)$ gives the exact sequence

$$
\begin{aligned}
\cdots \rightarrow \mathrm{H}^{1}\left(X_{\bar{k}}, \tilde{X}_{\bar{k}} ; R(1)\right) & \rightarrow \mathrm{H}^{1}\left(\tilde{X}_{\bar{k}}, R(1)\right) \rightarrow \mathrm{H}^{1}\left(X_{\bar{k}}, R(1)\right) \\
\rightarrow & \mathrm{H}^{2}\left(X_{\bar{k}}, \tilde{X}_{\bar{k}} ; R(1)\right) \rightarrow \mathrm{H}^{2}\left(\tilde{X}_{\bar{k}}, R(1)\right) \rightarrow \mathrm{H}^{2}\left(X_{\bar{k}}, R(1)\right) \rightarrow \cdots
\end{aligned}
$$

By purity [25, Chapter VI, Theorem 5.1], the relative cohomology groups are computed by

$$
\mathrm{H}^{1}\left(\tilde{X}_{\bar{k}}, X_{\bar{k}} ; R(1)\right) \cong 0 \quad \text { and } \quad \mathrm{H}^{2}\left(\tilde{X}_{\bar{k}}, X_{\bar{k}} ; R(1)\right) \cong \mathrm{H}^{0}\left(\partial X_{\bar{k}}, R\right) .
$$


By Poincaré duality [25, Chapter VI, Theorem 11.1], there is a unique isomorphism $\mathrm{H}^{2}\left(\tilde{X}_{\bar{k}}, R(1)\right) \rightarrow R$ taking the cycle class of a point to $1[9$, Cycle, 2.1.5, page 139]. Since $X_{\bar{k}}$ is affine of dimension 1 , the group $\mathrm{H}^{2}\left(X_{\bar{k}}, R(1)\right)$ vanishes. Substituting these computations into the above gives

$$
0 \longrightarrow \mathrm{H}^{1}\left(\tilde{X}_{\bar{k}}, R(1)\right) \longrightarrow \mathrm{H}^{1}\left(X_{\bar{k}}, R(1)\right) \longrightarrow \bigoplus_{\partial X(\bar{k})} R \stackrel{\oplus \mathrm{id}}{\longrightarrow} R \longrightarrow 0 .
$$

It is straightforward to check that $\theta$ induces a map of exact sequences from (24) with $\pi=\pi_{1}^{\ell}\left(\mathrm{Pic}^{-1} \tilde{X}_{\bar{k}}, \mathrm{Ab}_{*} \partial X(\bar{k})\right)$ to (27) which is the identity on $\bigoplus_{\partial X(\bar{k})} R$ and $R$. By (25), there is a map

$$
\pi_{1} \rightarrow \underset{n}{\lim } \operatorname{Pic}^{0} \tilde{X}(\bar{k})\left[\ell^{n}\right]
$$

which is an isomorphism because $\operatorname{Pic}^{0} \tilde{X}_{\bar{k}}$ is an abelian variety [27, Chapter IV, 18, Serre-Lang theorem]. Since the Néron-Severi group of $\tilde{X}_{\bar{k}}$ is torsion-free,

$$
\operatorname{Pic}^{0} \tilde{X}(\bar{k})\left[\ell^{n}\right] \cong \operatorname{Pic} \tilde{X}(\bar{k})\left[\ell^{n}\right] .
$$

By the moduli definition of the Picard functor, its $\ell^{n}$-torsion points over $\bar{k}$ are

$$
\operatorname{Pic} \tilde{X}(\bar{k})\left[\ell^{n}\right] \cong \mathrm{H}^{1}\left(\tilde{X}_{\bar{k}}, \mathbb{G}_{m}\right)\left[\ell^{n}\right] .
$$

The Kummer exact sequence

$$
1 \longrightarrow \mu_{\ell^{n}} \longrightarrow \mathbb{G}_{m} \stackrel{b \mapsto b^{\ell^{n}}}{\longrightarrow} \mathbb{G}_{m} \longrightarrow 1
$$

and the fact that $\bar{k}^{*}$ is $\ell^{n}$-divisible shows

$$
\mathrm{H}^{1}\left(\tilde{X}_{\bar{k}}, \mathbb{G}_{m}\right)\left[\ell^{n}\right] \cong \mathrm{H}^{1}\left(\tilde{X}_{\bar{k}}, \mu_{\ell^{n}}\right),
$$

whence

$$
\pi_{1} \cong \mathrm{H}^{1}\left(\tilde{X}_{\bar{k}}, \mathbb{Z}_{\ell}(1)\right)
$$

Thus $\theta$ is an isomorphism.

\section{Homology of $\overline{\text { Pic }} X^{+}$}

This section gives a canonical isomorphism of $\operatorname{Gal}(\bar{k} / k)$-modules

$$
\mathrm{H}_{1}\left(\overline{\mathrm{Pic}}^{-1} X_{\bar{k}}^{+}, R\right) \rightarrow \mathrm{H}^{1}\left(X_{\bar{k}}, R(1)\right) .
$$


By Theorem 3.19, there is a universal homeomorphism $\bar{P}^{\natural} \rightarrow \overline{\mathrm{P} i c}^{-1} X^{+}$, where $\bar{P}^{\natural}$ is given by the pushout square

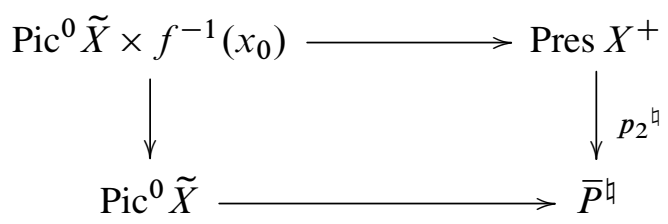

which after base change to $\bar{k}$ becomes the pushout square:

$$
\begin{aligned}
& \bigsqcup_{\partial X(\bar{k})} \operatorname{Pic}^{0} \tilde{X}_{\bar{k}} \stackrel{\hat{\epsilon}_{\bar{k}}}{\longrightarrow} \operatorname{Pres} X_{\bar{k}}^{+} \\
& \text {Ш id } \downarrow \\
& \operatorname{Pic}^{0} \tilde{X}_{\bar{k}} \longrightarrow \bar{P}_{\bar{k}}^{\natural}
\end{aligned}
$$

Assume $\tilde{X}_{\bar{k}}$ is not genus 0 . Let $e$ be a geometric point of $\operatorname{Pic}^{0} \tilde{X}_{\bar{k}}$ whose image is the identity element. The maps $\operatorname{Pic}^{0} \tilde{X}_{\bar{k}} \rightarrow$ Pres $X_{\bar{k}}^{+}$making up $\hat{\epsilon}_{\bar{k}}$ send $e$ to a set of geometric points of Pres $X_{\vec{k}}^{+}$, denoted $\mathcal{E}$. Since all points of $\mathcal{E}$ have the same image in $\bar{P}_{\bar{k}}^{\natural}$, there is an induced $\mathrm{Gal}(\bar{k} / k)$-equivariant map

$$
\pi_{1}^{\ell}\left(\operatorname{Pres} X_{\bar{k}}^{+}, \mathcal{E}\right) \rightarrow \pi_{1}^{\ell}\left(\bar{P}_{\bar{k}}^{\natural}\right) .
$$

Composing with the Hurewicz map gives $\pi_{1}^{\ell}\left(\operatorname{Pres} X_{\bar{k}}^{+}, \mathcal{E}\right) \rightarrow \mathrm{H}_{1}\left(\bar{P}_{\bar{k}}^{\natural}, R\right)$, whence

$$
\mathcal{F}_{R} \pi_{1}^{\ell}\left(\operatorname{Pres} X_{\bar{k}}^{+}, \mathcal{E}\right) \rightarrow \mathrm{H}_{1}\left(\bar{P}_{\bar{k}}^{\natural}, R\right)
$$

By Proposition 3.8, the presentation scheme is a projective bundle

$$
p_{1 \bar{k}}: \text { Pres } X_{\bar{k}}^{+} \rightarrow \operatorname{Pic}^{-1} \tilde{X}_{\bar{k}}
$$

such that the composite map

$$
\coprod_{\partial X(\bar{k})} \operatorname{Pic}^{0} \tilde{X}_{\bar{k}} \rightarrow \operatorname{Pres} X_{\bar{k}}^{+} \rightarrow \operatorname{Pic}^{-1} \tilde{X}_{\bar{k}}
$$

is the coproduct over $\tilde{x}$ in $\partial X(\bar{k})$ of the maps $L \mapsto L \otimes I_{\tilde{x}}$. This projective bundle induces a $\operatorname{Gal}(\bar{k} / k)$-equivariant isomorphism

$$
\pi_{1}^{\ell}\left(\operatorname{Pres} X_{\bar{k}}^{+}, \mathcal{E}\right) \rightarrow \pi_{1}^{\ell}\left(\operatorname{Pic}^{-1} \tilde{X}_{\bar{k}}, \mathrm{Ab}_{*} \partial X(\bar{k})\right)
$$

by the homotopy exact sequence for the fundamental group [17, Exposé X, Corollary 1.4], which extends to this isomorphism of groupoids, for instance by (24). Here 
we are using that the geometric points of $\mathrm{Ab}_{*} \partial X(\bar{k})$ are distinct, which follows from the hypothesis that $\widetilde{X}_{\bar{k}}$ is not genus 0 . Applying Theorem 5.1 defines

$$
\sigma: \mathrm{H}^{1}\left(X_{\bar{k}}, R(1)\right) \rightarrow \mathrm{H}_{1}\left(\bar{P}_{\bar{k}}^{\natural}, R\right)
$$

Proposition 6.1 $\sigma$ is an isomorphism.

Proof The Mayer-Vietoris sequence (see Appendix B) corresponding to (28) gives the exact sequence of $\operatorname{Gal}(\bar{k} / k)$-modules

$$
\begin{aligned}
& \bigoplus \bigoplus_{\partial X(\bar{k})} \mathrm{H}_{1}\left(\operatorname{Pic}^{0} \tilde{X}_{\bar{k}}, R\right) \rightarrow \mathrm{H}_{1}\left(\operatorname{Pic}^{0} \tilde{X}_{\bar{k}}, R\right) \bigoplus \mathrm{H}_{1}\left(\operatorname{Pres} X_{\bar{k}}^{+}, R\right) \rightarrow \mathrm{H}_{1}\left(\bar{P}_{\bar{k}}^{\natural}, R\right) \\
& \quad \rightarrow \bigoplus_{\partial X(\bar{k})} \mathrm{H}_{0}\left(\operatorname{Pic}^{0} \tilde{X}_{\bar{k}}, R\right) \rightarrow \mathrm{H}_{0}\left(\operatorname{Pic}^{0} \tilde{X}_{\bar{k}}, R\right) \bigoplus \mathrm{H}_{0}\left(\operatorname{Pres} X_{\bar{k}}^{+}, R\right) \rightarrow \mathrm{H}_{0}\left(\bar{P}_{\bar{k}}^{\natural}, R\right)
\end{aligned}
$$

by Theorem B.4.

Let $*=0$ or 1 . Applying $\mathrm{H}_{*}$ to $\hat{\epsilon}_{\bar{k}}$ is the $\partial X(\bar{k})$-fold coproduct of a fixed isomorphism, as can be seen by noting that $\mathrm{H}_{*}\left(\right.$ Pres $\left.X_{\bar{k}}^{+}, R\right) \rightarrow \mathrm{H}_{*}\left(\mathrm{Pic}^{-1} \tilde{X}_{\bar{k}}, R\right)$ is an isomorphism since Pres $X_{\bar{k}}^{+} \rightarrow \operatorname{Pic}^{-1} \tilde{X}_{\bar{k}}$ is a projective bundle, and

$$
\mathrm{H}_{*}\left(L \mapsto L \otimes I_{\tilde{x}}: \operatorname{Pic}^{0} \tilde{X}_{\bar{k}} \rightarrow \operatorname{Pic}^{-1} \tilde{X}_{\bar{k}}\right)
$$

is an isomorphism.

This gives the exact sequence

$0 \rightarrow \mathrm{H}_{1}\left(\operatorname{Pres} X_{\bar{k}}^{+}, R\right) \rightarrow \mathrm{H}_{1}\left(\bar{P}_{\bar{k}}^{\natural}, R\right) \rightarrow \bigoplus_{\partial X(\bar{k})} \mathrm{H}_{0}\left(\operatorname{Pic}^{0} \tilde{X}_{\bar{k}}, R\right) \rightarrow \mathrm{H}_{0}\left(\operatorname{Pic}^{0} \tilde{X}_{\bar{k}}, R\right) \rightarrow 0$,

where the map

$$
\bigoplus_{\partial X(\bar{k})} \mathrm{H}_{0}\left(\operatorname{Pic}^{0} \tilde{X}_{\bar{k}}, R\right) \rightarrow \mathrm{H}_{0}\left(\operatorname{Pic}^{0} \tilde{X}_{\bar{k}}, R\right)
$$

is the $\partial X(\bar{k})$-fold coproduct of the identity map.

Identifying $\mathrm{H}_{0}\left(\operatorname{Pic}^{0} \tilde{X}_{\bar{k}}, R\right)$ with $R$, we obtain

$$
0 \rightarrow \mathrm{H}_{1}\left(\operatorname{Pres} X_{\bar{k}}^{+}, R\right) \rightarrow \mathrm{H}_{1}\left(\bar{P}_{\bar{k}}^{\natural}, R\right) \rightarrow \bigoplus_{\partial X(\bar{k})} R \rightarrow R \rightarrow 0 .
$$

By (30), the definition of the Mayer-Vietoris sequence and (24), it follows that (29) is an isomorphism, proving the proposition. 
If $\tilde{X}_{\bar{k}}$ is genus 0 , we have that $\operatorname{Pic}^{0} \tilde{X}_{\bar{k}} \cong \operatorname{Spec} \bar{k}$. By the Mayer-Vietoris sequence (Theorem B.4) corresponding to (28), we have an exact sequence

$$
0 \rightarrow \mathrm{H}_{1}\left(\operatorname{Pres} X_{\bar{k}}^{+}, R\right) \rightarrow \mathrm{H}_{1}\left(\overline{\mathrm{P}_{i c}}{ }^{-1} X_{\bar{k}}^{+}, R\right) \rightarrow \bigoplus_{\partial X(\bar{k})} R \rightarrow R \rightarrow 0 .
$$

By Proposition 3.8, the presentation scheme is a projective bundle over $\operatorname{Spec} \bar{k}$, whence $\mathrm{H}_{1}\left(\operatorname{Pres} X_{\bar{k}}^{+}, R\right)=0$, giving the desired isomorphism in this case by Remark 5.3.

\section{The Abel map gives Poincaré duality}

In this section we prove the main theorem, namely that the Altman-Kleiman Abel map realizes Poincaré duality.

Let $\operatorname{Tr}: \mathrm{H}^{2}\left(X_{\bar{k}}^{+}, R(1)\right) \cong \mathrm{H}_{c}^{2}\left(X_{\bar{k}}, R(1)\right) \rightarrow R$ denote the trace map, sending the class of a point to 1 [9, Cycle, 2.1.5, page 139].

Let $\wp: \mathrm{H}_{1}\left(X_{\bar{k}}^{+}, R\right) \rightarrow \mathrm{H}^{1}\left(X_{\bar{k}}, R(1)\right)$ be the Poincaré duality isomorphism characterized by $\operatorname{Tr}(\gamma \cup \wp(\lambda))=\langle\lambda, \gamma\rangle$ for all $\lambda$ in $\mathrm{H}_{1}\left(X_{\bar{k}}^{+}, R\right)$ and $\gamma$ in $\mathrm{H}^{1}\left(X_{\bar{k}}^{+}, R\right) \cong$ $\mathrm{H}_{c}^{1}\left(X_{\bar{k}}, R\right)$, where $\langle-,-\rangle$ denotes the tautological pairing between $\mathrm{H}_{1}$ and $\mathrm{H}^{1}$, defined as follows: if $l$ in $\pi_{1}^{\ell}\left(X_{\bar{k}}^{+}\right)$represents $\lambda$, then $l$ acts by addition by $\langle\lambda, \gamma\rangle$ on the fiber of the torsor classified by $\gamma$.

\section{Theorem 7.1}

$$
-\wp=\sigma^{-1} \mathrm{H}_{1}\left(\mathrm{Ab}_{\bar{k}}\right)
$$

To prove Theorem 7.1, we equip ourselves with three lemmas.

For a product $Y \times Z$, let $\mathrm{pr}_{1}: Y \times Z \rightarrow Y$ and $\mathrm{pr}_{2}: Y \times Z \rightarrow Z$ denote the projections. Let $N$ be a positive integer not divisible by the characteristic of $k$.

Let $g: X_{\bar{k}} \rightarrow \tilde{X}_{\bar{k}}$ denote the open immersion, resulting in another open immersion

$$
g \times 1: X_{\bar{k}} \times X_{\bar{k}} \rightarrow \tilde{X}_{\bar{k}} \times X_{\bar{k}} .
$$

The diagonal $\Delta$ of $X_{\bar{k}} \times X_{\bar{k}}$ defines a class $\operatorname{cl}(\Delta)$ in $\mathrm{H}_{\Delta}^{2}\left(X_{\bar{k}} \times X_{\bar{k}}, \mu_{N}\right)$ by [25, Chapter VI, Section 6, Theorem 6.1]. Furthermore, $\Delta$ is closed in $\tilde{X}_{\bar{k}} \times X_{\bar{k}}$, allowing us to apply excision [25, Chapter III, Section 1, Proposition 1.27] which defines an isomorphism $\mathrm{H}_{\Delta}^{2}\left(X_{\bar{k}} \times X_{\bar{k}}, \mu_{N}\right) \cong \mathrm{H}_{\Delta}^{2}\left(\tilde{X}_{\bar{k}} \times X_{\bar{k}},(g \times 1) ! \mu_{N}\right)$.

The adjunction $(g \times 1) !,(g \times 1)^{*}$ and the map $\mu_{N} \rightarrow \operatorname{pr}_{1}^{*} g^{*} g_{!} \mu_{N} \cong(g \times 1)^{*} \operatorname{pr}_{1}^{*} g ! \mu_{N}$ on $X_{\bar{k}} \times X_{\bar{k}}$ define a map $(g \times 1) ! \mu_{N} \rightarrow \operatorname{pr}_{1}^{*}\left(g_{!} \mu_{N}\right)$, which is an isomorphism because it induces isomorphisms on all stalks. The isomorphisms

$$
\begin{aligned}
(g \times 1) ! \mu_{N} \cong \operatorname{pr}_{1}^{*}\left(g_{!} \mu_{N}\right) \cong \operatorname{pr}_{1}^{*}\left(g_{!} \mathbb{Z} / N \otimes \mu_{N}\right) & \cong \operatorname{pr}_{1}^{*}\left(g_{!} \mathbb{Z} / N\right) \otimes \mu_{N} \\
& \cong \operatorname{pr}_{1}^{*}(g ! \mathbb{Z} / N) \otimes \operatorname{pr}_{2}^{*} \mu_{N}
\end{aligned}
$$


allow us to apply the Künneth formula to $\mathrm{H}^{*}\left(\tilde{X}_{\bar{k}} \times X_{\bar{k}},(g \times 1) ! \mu_{N}\right)$, from which we obtain

$$
\begin{aligned}
\mathrm{H}^{*}\left(\tilde{X}_{\bar{k}} \times X_{\bar{k}},(g \times 1) ! \mu_{N}\right) & \cong \mathrm{H}^{*}\left(\tilde{X}_{\bar{k}}, g ! \mathbb{Z} / n\right) \otimes \mathrm{H}^{*}\left(X_{\bar{k}}, \mu_{N}\right) \\
& \cong \mathrm{H}_{c}^{*}\left(X_{\bar{k}}, \mathbb{Z} / N\right) \otimes \mathrm{H}^{*}\left(X_{\bar{k}}, \mu_{N}\right) .
\end{aligned}
$$

This allows us to speak of $(i, j)$ Künneth components of elements of

$$
\mathrm{H}^{*}\left(\tilde{X}_{\bar{k}} \times X_{\bar{k}},(g \times 1) ! \mu_{N}\right) .
$$

Let $c^{1,1}$ be the $(1,1)$ Künneth component of the image of $\operatorname{cl}(\Delta)$ under

$$
\mathrm{H}_{\Delta}^{2}\left(\tilde{X}_{\bar{k}} \times X_{\bar{k}},(g \times 1) ! \mu_{N}\right) \rightarrow \mathrm{H}^{2}\left(\tilde{X}_{\bar{k}} \times X_{\bar{k}},(g \times 1) ! \mu_{N}\right) .
$$

We may view $c^{1,1}$ as an element of

$$
\mathrm{H}_{c}^{1}\left(X_{\bar{k}}, \mathrm{H}^{1}\left(X_{\bar{k}}, \mu_{N}\right)\right) \cong \mathrm{H}^{1}\left(\tilde{X}_{\bar{k}}, g_{!} \mathbb{Z} / N\right) \otimes \mathrm{H}^{1}\left(X_{\bar{k}}, \mu_{N}\right) .
$$

The diagonal $\widetilde{\Delta}$ of $\tilde{X}_{\bar{k}} \times \tilde{X}_{\bar{k}}$ determines a class

$$
\operatorname{cl}(\tilde{\Delta}) \in \mathrm{H}_{\widetilde{\Delta}}^{2}\left(\tilde{X}_{\bar{k}} \times \tilde{X}_{\bar{k}}, \mu_{N}\right) .
$$

Let $d^{1,1}$ denote the $(1,1)$ Künneth component of the image of $\operatorname{cl}(\widetilde{\Delta})$ under

$$
\mathrm{H}_{\widetilde{\Delta}}^{2}\left(\tilde{X}_{\bar{k}} \times \tilde{X}_{\bar{k}}, \operatorname{pr}_{1}^{*} \mathbb{Z} / N \otimes \operatorname{pr}_{2}^{*} \mu_{N}\right) \rightarrow \mathrm{H}^{2}\left(\tilde{X}_{\bar{k}} \times \tilde{X}_{\bar{k}}, \operatorname{pr}_{1}^{*} \mathbb{Z} / N \otimes \operatorname{pr}_{2}^{*} \mu_{N}\right),
$$

which we view as an element of $\mathrm{H}^{1}\left(\tilde{X}_{\bar{k}}, \mathrm{H}^{1}\left(\tilde{X}_{\bar{k}}, \mu_{N}\right)\right)$.

Since there is a map of smooth pairs $\left(\Delta, X_{\bar{k}} \times X_{\bar{k}}\right) \rightarrow\left(\widetilde{\Delta}, \tilde{X}_{\bar{k}} \times \tilde{X}_{\bar{k}}\right)$, we have

$$
(g \times g)^{*} \operatorname{cl}(\tilde{\Delta})=\operatorname{cl}(\Delta)
$$

by [25, Chapter VI, Section 6, Theorem 6.1(c)].

Our first lemma rewrites (31) in terms of $f: \tilde{X} \rightarrow X^{+}$. The viewpoint is that

$$
\mathrm{H}_{c}^{1}\left(X_{\bar{k}}, \mathrm{H}^{1}\left(X_{\bar{k}}, \mu_{N}\right)\right) \text { and } \mathrm{H}^{1}\left(\tilde{X}_{\bar{k}}, \mathrm{H}^{1}\left(\tilde{X}_{\bar{k}}, \mu_{N}\right)\right)
$$

classify certain torsors on $X_{\bar{k}}^{+}$and $\tilde{X}_{\bar{k}}$, respectively, and (31) computes in terms of $d^{1,1}$ the pullback under $f$ of the torsor classified by $c^{1,1}$.

To describe pullback by $f: \tilde{X} \rightarrow X^{+}$more specifically, note that $f$ induces

$$
f^{*}: \mathrm{H}^{1}\left(X_{\bar{k}}^{+}, \mathrm{H}^{1}\left(X_{\bar{k}}, \mu_{N}\right)\right) \cong \mathrm{H}_{c}^{1}\left(X_{\bar{k}}, \mathrm{H}^{1}\left(X_{\bar{k}}, \mu_{N}\right)\right) \rightarrow \mathrm{H}^{1}\left(\tilde{X}_{\bar{k}}, \mathrm{H}^{1}\left(X_{\bar{k}}, \mu_{N}\right)\right) .
$$

Equivalently, tensoring the map $g ! \mathbb{Z} / N \rightarrow \mathbb{Z} / N$ of sheaves on $\tilde{X}$ with $\mathrm{H}^{1}\left(X_{\bar{k}}, \mu_{N}\right)$ and applying $\mathrm{H}^{1}\left(\tilde{X}_{\bar{k}},-\right)$ gives $f^{*}$. 
We introduce one last piece of notation. The open immersion $g: X \rightarrow \tilde{X}$ induces a map

$$
g^{*}: \mathrm{H}^{1}\left(\tilde{X}_{\bar{k}}, \mu_{N}\right) \rightarrow \mathrm{H}^{1}\left(X_{\bar{k}}, \mu_{N}\right),
$$

and applying $\mathrm{H}^{1}\left(\widetilde{X}_{\bar{k}},-\right)$ gives a map

$$
\mathrm{H}^{1}\left(\tilde{X}_{\bar{k}}, g^{*}\right): \mathrm{H}^{1}\left(\tilde{X}_{\bar{k}}, \mathrm{H}^{1}\left(\tilde{X}_{\bar{k}}, \mu_{N}\right)\right) \rightarrow \mathrm{H}^{1}\left(\tilde{X}_{\bar{k}}, \mathrm{H}^{1}\left(X_{\bar{k}}, \mu_{N}\right)\right) \text {. }
$$

\section{Lemma 7.2}

$$
\mathrm{H}^{1}\left(\tilde{X}_{\bar{k}}, g^{*}\right) d^{1,1}=f^{*} c^{1,1}
$$

Proof We may assume $k=\bar{k}$. The following diagram is commutative: the top two rows commute by commutativity of boundary maps associated to the map of pairs

$$
(\Delta, X \times X) \rightarrow(\tilde{\Delta}, \tilde{X} \times \tilde{X}) ;
$$

the second and third rows by naturality of the Künneth formula; the right trapezoid also by naturality of the Künneth formula; and the bottom triangle obviously:

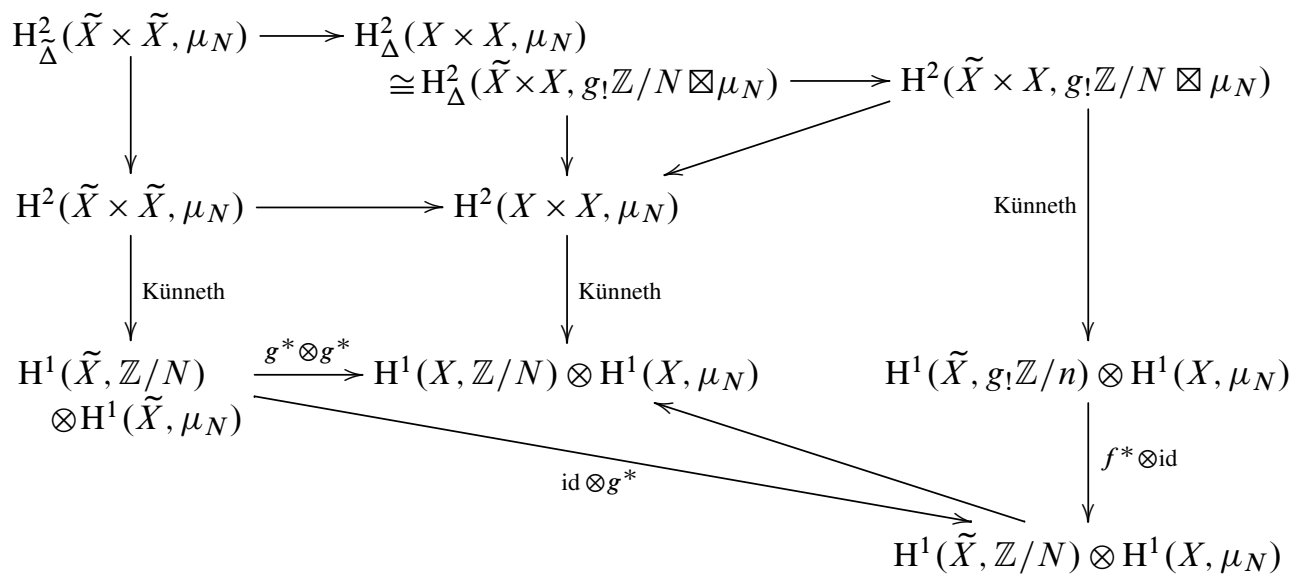

Here the notation $(-) \otimes(-)$ is an abbreviation for $\operatorname{pr}_{1}^{*}(-) \otimes \operatorname{pr}_{2}^{*}(-)$.

The image of $\operatorname{cl}(\tilde{\Delta}) \in \mathrm{H}_{\tilde{\Delta}}^{2}\left(\tilde{X} \times \tilde{X}, \mu_{N}\right)$ under the top two horizontal morphisms followed by the right vertical morphism is $f^{*} c^{1,1}$. The image of $\operatorname{cl}(\tilde{\Delta})$ under the left two vertical morphisms followed by the bottom diagonal morphism is $\mathrm{H}^{1}\left(\tilde{X}, g^{*}\right) d^{1,1}$, showing the proposition.

The next lemma is a close analogue of [9, Dualité, Proposition 3.4, page 164], whose proof is almost identical, but we include it for completeness.

The trace map $\operatorname{Tr}: \mathrm{H}_{c}^{2}\left(X_{\bar{k}}, \mu_{N}\right) \rightarrow \mathbb{Z} / N$ defines a map

$$
\operatorname{Tr}^{\prime}: \mathrm{H}^{1}\left(\tilde{X}_{\bar{k}}, g_{!} \mathbb{Z} / N\right) \otimes \mathrm{H}^{1}\left(X_{\bar{k}}, \mu_{N}\right) \otimes \mathrm{H}^{1}\left(X_{\bar{k}}, \mu_{N}\right) \rightarrow \mathrm{H}^{1}\left(X_{\bar{k}}, \mu_{N}\right)
$$


given by

$$
a \otimes b \otimes c \mapsto \operatorname{Tr}(a \cup c) b .
$$

Lemma 7.3 For all $x \in \mathrm{H}^{1}\left(X_{\bar{k}}, \mu_{N}\right)$, we have $\operatorname{Tr}^{\prime}\left(-c^{1,1} \otimes x\right)=x$.

Proof We may assume $k=\bar{k}$. The above isomorphism $(g \times 1) ! \mu_{N} \cong \operatorname{pr}_{1}^{*} g_{!} \mu_{N}$ induces an isomorphism $(g \times 1) ! \mu_{N} \otimes \mu_{N} \cong g_{!} \mu_{N} \otimes \mu_{N}$ of sheaves on $\tilde{X} \times X$. We obtain a Künneth formula for $\mathrm{H}^{*}\left(\tilde{X} \times X,(g \times 1) ! \mu_{N} \otimes \mu_{N}\right) \cong \mathrm{H}^{*}\left(\tilde{X}, g_{!} \mu_{N}\right) \otimes \mathrm{H}^{*}\left(X, \mu_{N}\right)$, allowing us to define $\operatorname{Tr}_{3}$ to be the composition

$$
\begin{aligned}
\operatorname{Tr}_{3}: \mathrm{H}^{3}\left(\tilde{X} \times X,(g \times 1) ! \mu_{N} \otimes \mu_{N}\right) & \cong \mathrm{H}^{3}\left(\tilde{X} \times X, g_{!} \mu_{N} \otimes \mu_{N}\right) \\
& \longrightarrow \mathrm{H}^{2}\left(\tilde{X}, g_{!} \mu_{N}\right) \otimes \mathrm{H}^{1}\left(X, \mu_{N}\right) \longrightarrow \mathrm{H}^{1}\left(X, \mu_{N}\right)
\end{aligned}
$$

which takes the $(2,1)$ Künneth component and then applies trace.

There is a cup product [9, Cycle, 1.2.4, page 134]

$$
\mathrm{H}^{2}\left(\tilde{X} \times X,(g \times 1) ! \mu_{N}\right) \times \mathrm{H}^{1}\left(X \times X, \mu_{N}\right) \rightarrow \mathrm{H}^{3}\left(\tilde{X} \times X,(g \times 1) ! \mu_{N} \otimes \mu_{N}\right) .
$$

Note that

$$
\operatorname{Tr}^{\prime}\left(-c^{1,1} \otimes x\right)=-\operatorname{Tr}_{3}\left(-c^{1,1} \cup \operatorname{pr}_{1}^{*} x\right)=\operatorname{Tr}_{3}\left(c^{1,1} \cup \operatorname{pr}_{1}^{*} x\right),
$$

where the minus sign after the first equality comes from permuting cochains of degree 1 in (32).

Since $c^{1,1} \cup \operatorname{pr}_{1}^{*} x$ and $\operatorname{cl}(\Delta) \cup \operatorname{pr}_{1}^{*} x$ have the same $(2,1)$ Künneth component,

$$
\operatorname{Tr}_{3}\left(c^{1,1} \cup \operatorname{pr}_{1}^{*} x\right)=\operatorname{Tr}_{3}\left(\operatorname{cl}(\Delta) \cup \operatorname{pr}_{1}^{*} x\right) .
$$

The cup product on the right-hand side may be reinterpreted as the cup product

$$
\mathrm{H}_{\Delta}^{2}\left(\tilde{X} \times X,(g \times 1) ! \mu_{N}\right) \times \mathrm{H}^{1}\left(\Delta, \mu_{N}\right) \rightarrow \mathrm{H}_{\Delta}^{3}\left(\tilde{X} \times X,(g \times 1) ! \mu_{N} \otimes \mu_{N}\right)
$$

defined in [9, Cycle, 1.2.2.2, page 133].

Since $\operatorname{pr}_{1}=\operatorname{pr}_{2}$ when restricted to $\Delta$, we therefore have

$$
\operatorname{Tr}_{3}\left(\operatorname{cl}(\Delta) \cup \operatorname{pr}_{1}^{*} x\right)=\operatorname{Tr}_{3}\left(\operatorname{cl}(\Delta) \cup \operatorname{pr}_{2}^{*} x\right)=\operatorname{Tr}_{4}(\operatorname{cl}(\Delta)) \cup x=x,
$$

where

$$
\operatorname{Tr}_{4}: \mathrm{H}^{2}\left(\tilde{X} \times X,(g \times 1) ! \mu_{N}\right) \cong \mathrm{H}^{2}\left(\tilde{X} \times X, g_{!} \mu_{N} \otimes \mathbb{Z} / N\right) \rightarrow \mathbb{Z} / N
$$

takes the $(2,0)$ Künneth component and applies Tr.

Combining (33), (34), and (35) completes the proof. 
Recall the notation $\mathcal{F}_{R}$ for the free $R$-module on a groupoid from Section 5. The next lemma identifies $\mathrm{H}_{1}\left(X_{\bar{k}}^{+}, R\right)$ in terms of the free $R$-module associated to paths in $\widetilde{X}_{\bar{k}}$ between geometric points of $\partial X$.

Lemma 7.4 The map $\mathcal{F}_{R} \pi_{1}^{\ell}\left(\tilde{X}_{\bar{k}}, \partial X(\bar{k})\right) \rightarrow \mathrm{H}_{1}\left(X_{\bar{k}}^{+}, R\right)$ induced by

$$
\pi_{1}^{\ell}\left(\tilde{X}_{\bar{k}}, \partial X(\bar{k})\right) \stackrel{f_{*}}{\longrightarrow} \pi_{1}^{\ell}\left(X_{\bar{k}}^{+}, \bar{x}_{0}\right) \longrightarrow \mathrm{H}_{1}\left(X_{\bar{k}}^{+}, R\right)
$$

is an isomorphism.

Proof The Mayer-Vietoris sequence (Theorem B.4 of Appendix B) corresponding to

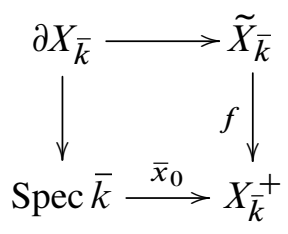

gives the exact sequence of $\operatorname{Gal}(\bar{k} / k)$-modules

$\bigoplus \mathrm{H}_{1}(\operatorname{Spec} \bar{k}, R) \rightarrow \mathrm{H}_{1}(\operatorname{Spec} \bar{k}, R) \oplus \mathrm{H}_{1}\left(\tilde{X}_{\bar{k}}, R\right) \rightarrow \mathrm{H}_{1}\left(X_{\bar{k}}^{+}, R\right)$

$\partial X(\bar{k})$

$$
\rightarrow \bigoplus_{\partial X(\bar{k})} \mathrm{H}_{0}(\operatorname{Spec} \bar{k}, R) \rightarrow \mathrm{H}_{0}(\operatorname{Spec} \bar{k}, R) \oplus \mathrm{H}_{0}\left(\tilde{X}_{\bar{k}}, R\right) \rightarrow \mathrm{H}_{0}\left(X_{\bar{k}}^{+}, R\right),
$$

which we can rewrite as

$$
0 \rightarrow \mathrm{H}_{1}\left(\tilde{X}_{\bar{k}}, R\right) \rightarrow \mathrm{H}_{1}\left(X_{\bar{k}}^{+}, R\right) \rightarrow \bigoplus_{\partial X(\bar{k})} R \rightarrow R \rightarrow 0
$$

using the isomorphisms $\mathrm{H}_{1}(\operatorname{Spec} \bar{k}, R)=0, \mathrm{H}_{0}(\operatorname{Spec} \bar{k}, R) \cong \mathrm{H}_{0}\left(\tilde{X}_{\bar{k}}, R\right) \cong R$, and noting that the map

$$
\bigoplus_{\partial X(\bar{k})} \mathrm{H}_{0}(\operatorname{Spec} \bar{k}, R) \rightarrow \mathrm{H}_{0}(\operatorname{Spec} \bar{k}, R) \oplus \mathrm{H}_{0}\left(\tilde{X}_{\bar{k}}, R\right)
$$

is identified with the coproduct over $\partial X(\bar{k})$ of the diagonal map $R \rightarrow R \oplus R$.

Associated to $\mathcal{F}_{R} \pi_{1}^{\ell}\left(\tilde{X}_{\bar{k}}, \partial X\right)$ is the exact sequence (24)

$$
0 \rightarrow \mathcal{F}_{R} \pi_{1}^{\ell}\left(\tilde{X}_{\bar{k}}, \bar{x}_{0}\right) \rightarrow \mathcal{F}_{R} \pi_{1}^{\ell}\left(\tilde{X}_{\bar{k}}, \partial X\right) \rightarrow \oplus_{\partial X_{\bar{k}}} R \rightarrow R \rightarrow 0,
$$


which is compatible with the Mayer-Vietoris sequence in the sense that the diagram

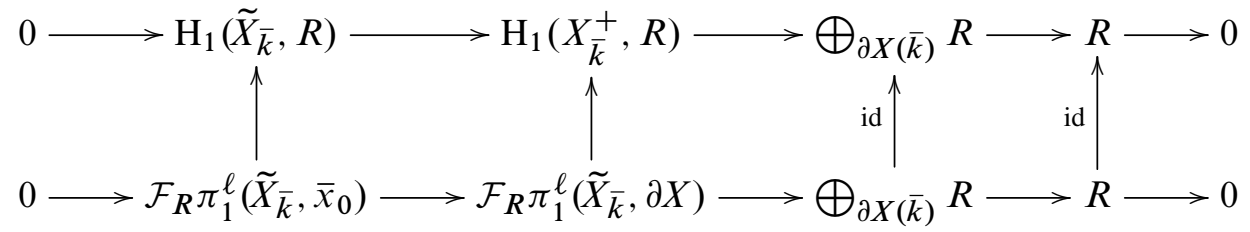

commutes.

Since $\mathcal{F}_{R} \pi_{1}^{\ell}\left(\tilde{X}_{\bar{k}}, \bar{x}_{0}\right) \rightarrow \mathrm{H}_{1}\left(\tilde{X}_{\bar{k}}, R\right)$ is an isomorphism, $\mathcal{F}_{R} \pi_{1}^{\ell}\left(\tilde{X}_{\bar{k}}, \partial X\right) \rightarrow \mathrm{H}_{1}\left(X_{\bar{k}}^{+}, R\right)$ is also an isomorphism.

We now give the proof of Theorem 7.1.

Proof of Theorem 7.1 Suppose $R=\mathbb{Z} / \ell^{n}$, and let $N=\ell^{n}$. By definition of the cohomology of an $\ell$-adic sheaf [25, pages 163-164], it suffices to prove the theorem in this case.

Since $\wp, \sigma$, and $\mathrm{H}_{1}\left(\mathrm{Ab}_{\bar{k}}\right)$ are $R$-module morphisms, by Lemma 7.4 it suffices to show that

$$
-\wp\left(f_{*} \gamma\right)=\sigma^{-1} \mathrm{H}_{1}\left(\mathrm{Ab}_{\bar{k}}\right)\left(f_{*} \gamma\right)
$$

for $\gamma \in \pi_{1}^{\ell}\left(\tilde{X}_{\bar{k}}, \partial X\right)$.

By Lemma 7.3,

$$
-\wp\left(f_{*} \gamma\right)=\operatorname{Tr}^{\prime}\left(c^{1,1} \otimes \wp\left(f_{*} \gamma\right)\right)
$$

By the definition of $\wp$,

$$
\operatorname{Tr}^{\prime}\left(c^{1,1} \otimes \wp\left(f_{*} \gamma\right)\right)=\left\langle f_{*} \gamma, c^{1,1}\right\rangle .
$$

The element $c^{1,1}$ classifies a $\mathrm{H}^{1}\left(X_{\bar{k}}, R(1)\right)$-torsor, which we will denote $Y \rightarrow X_{\bar{k}}^{+}$. As in Section 5, let $\phi_{x}(T)$ denote the fiber of a torsor $T$ at a geometric point $x$. Pulling back $Y$ by $f$ gives a $\mathrm{H}^{1}\left(X_{\bar{k}}, R(1)\right)$-torsor $f^{*} Y$ with isomorphisms $\phi_{\tilde{x}} f^{*} Y \cong \phi_{\bar{x}_{0}} Y$ for all $\tilde{x} \in \partial X(\bar{k})$, allowing us to speak of the monodromy of $f^{*} Y$ along $\gamma$ as an element $\left\langle\gamma, f^{*} c^{1,1}\right\rangle$ in $\mathrm{H}^{1}\left(X_{\bar{k}}, R(1)\right)$. Furthermore,

$$
\left\langle f_{*} \gamma, c^{1,1}\right\rangle=\left\langle\gamma, f^{*} c^{1,1}\right\rangle .
$$

By Lemma 7.2, $f^{*} Y$ is classified by $\mathrm{H}^{1}\left(\tilde{X}_{\bar{k}}, g^{*}\right) d^{1,1}$ in $\mathrm{H}^{1}\left(\tilde{X}_{\bar{k}}, \mathrm{H}^{1}\left(X_{\bar{k}}, R(1)\right)\right)$, which allows us to write

$$
\left\langle\gamma, f^{*} c^{1,1}\right\rangle=\left\langle\gamma, \mathrm{H}^{1}\left(\tilde{X}_{\bar{k}}, g^{*}\right) d^{1,1}\right\rangle,
$$

giving

$$
-\wp\left(f_{*} \gamma\right)=\left\langle\gamma, \mathrm{H}^{1}\left(\tilde{X}_{\bar{k}}, g^{*}\right) d^{1,1}\right\rangle
$$


by combining with the three previous equations.

Now consider $\sigma^{-1} \mathrm{H}_{1}\left(\mathrm{Ab}_{\bar{k}}\right)\left(f_{*} \gamma\right)$. By the construction of the lifted Abel map (see Definition 4.4),

$$
p_{2} \circ \operatorname{Pres}(\mathrm{Ab})=\mathrm{Ab} \circ f,
$$

whence $\mathrm{H}_{1}\left(\mathrm{Ab}_{\bar{k}}\right)\left(f_{*} \gamma\right)$ is the element of homology represented by

$$
p_{2 *} \circ \operatorname{Pres}(\mathrm{Ab})_{*} \gamma \in \pi_{1}^{\ell}\left(\overline{\mathrm{Pic}}^{-1} X^{+}, \mathrm{Ab}_{*} \bar{x}_{0}\right) .
$$

Consider an element of the homology group $\mathrm{H}_{1}\left(\overline{\mathrm{Pic}}^{-1} X_{\bar{k}}^{+}, R\right)$ represented by an element of $\pi_{1}^{\ell}\left(\overline{\mathrm{Pic}}^{-1} X_{\bar{k}}^{+}, \mathrm{Ab}_{*} \bar{x}_{0}\right)$ which is the image under $p_{2}$ of a path in Pres $X_{\bar{k}}^{+}$. The image under $\sigma^{-1}$ of such an element is particularly easy to evaluate. Here is the resulting description of $\sigma^{-1} p_{2 *} \circ \operatorname{Pres}(\mathrm{Ab})_{*} \gamma$. As in the proof of Theorem 5.1, let $a$ be a geometric point of $\operatorname{Pic}^{1} \tilde{X}_{\bar{k}}$, defining $+_{a}: \operatorname{Pic}^{-1} \tilde{X}_{\bar{k}} \rightarrow \operatorname{Pic}^{0} \tilde{X}_{\bar{k}}$. Let $m_{N}$ : $\operatorname{Pic}^{0} \tilde{X}_{\bar{k}} \rightarrow$ $\operatorname{Pic}^{0} \tilde{X}_{\bar{k}}$ denote the finite étale cover given by multiplication by $N$. Let $\tilde{x}_{s}$ and $\tilde{x}_{t}$ in $\partial X(\bar{k})$ be the source and target of $\gamma$. Since $\gamma$ is a path, $\gamma$ induces a morphism between fibers

$$
\phi_{\tilde{x}_{s}}\left(\mathrm{Ab}^{*}+{ }_{a}^{*} m_{N}\right) \rightarrow \phi_{\tilde{x}_{t}}\left(\mathrm{Ab}^{*}+{ }_{a}^{*} m_{N}\right),
$$

which is given by addition by an element $\left\langle\gamma, \mathrm{Ab}^{*}+{ }_{a}^{*} m_{N}\right\rangle$ of $\phi_{\mathrm{Ab} *} \tilde{x}_{t}-\mathrm{Ab}_{*} \tilde{x}_{s}\left[m_{N}\right]$. By construction, $\sigma^{-1} p_{2 *}\left(\operatorname{Pres}(\mathrm{Ab})_{*} \gamma\right)$ is the image under (26) of $\left\langle\gamma, \mathrm{Ab}^{*}+{ }_{a}^{*} m_{N}\right\rangle$.

By [9, Dualité, Proposition 3.2, page 162], $d^{1,1}$ classifies the pullback of $m_{N}$ by $+{ }_{a} \circ \mathrm{Ab}$. (The notation in [9] is that $u$ is the pullback of $m_{N}$ by the negative of $+{ }_{a} \circ \mathrm{Ab}$, whence the appearance of the sign.) Thus the torsor classified by $\mathrm{H}^{1}\left(\widetilde{X}_{\bar{k}}, g^{*}\right) d^{1,1}$ is

$$
\left(\mathrm{Ab}^{*}+{ }_{a}^{*} m_{N}\right) \times_{\mathrm{H}^{1}\left(\tilde{X}_{\bar{k}}, R(1)\right)} \mathrm{H}^{1}\left(X_{\bar{k}}, R(1)\right),
$$

which identifies the action of $\gamma$ on the fibers of $\mathrm{H}^{1}\left(\tilde{X}_{\bar{k}}, g^{*}\right) d^{1,1}$ with its action on the fibers of $d^{1,1}$. In particular, the map induced by $\gamma$ from the fiber of $\mathrm{H}^{1}\left(\tilde{X}_{\bar{k}}, g^{*}\right) d^{1,1}$ over $\tilde{x}_{s}$ to the fiber over $\tilde{x}_{t}$ is addition by $\left\langle\gamma, \mathrm{Ab}^{*}+{ }_{a}^{*} m_{N}\right\rangle$. The isomorphisms $\phi_{\tilde{x}_{s}} f^{*} Y \cong \phi_{\tilde{x}_{t}} f^{*} Y$ are compatible with (26), giving that the image of $\left\langle\gamma, \mathrm{Ab}^{*}+{ }_{a}^{*} m_{N}\right\rangle$ under (26) is $\left\langle\gamma, \mathrm{H}^{1}\left(\tilde{X}_{\bar{k}}, g^{*}\right) d^{1,1}\right\rangle$. Combining with (36) completes the proof.

\section{Appendix A: Cohomology in terms of the fundamental group of Pic}

In this appendix we prove Proposition 1.8 , identifying $\mathrm{H}^{1}\left((-)_{\bar{k}}, \mathbb{Z}_{\ell}(1)\right)$ with the $\ell-$ étale fundamental group of the Picard scheme, as well as Proposition A.4, giving a similar description of $\mathrm{H}^{1}\left((-)_{\bar{k}}, \mathbb{Z} / N(1)\right)$. 
Proposition 1.8 Let $k$ be a perfect field. There is a natural isomorphism of functors from proper, geometrically connected schemes over $k$ to $\mathrm{Gal}(\bar{k} / k)$-modules

$$
\pi_{1}^{\ell}\left(\operatorname{Pic}^{0}(-)_{\bar{k}}, e\right) \cong \mathrm{H}^{1}\left((-)_{\bar{k}}, \mathbb{Z}_{\ell}(1)\right),
$$

for $\ell$ a prime not equal to the characteristic of $k$.

Remark A.1 In the statement of Proposition 1.8, the $\ell$-étale fundamental group $\pi_{1}^{\ell}$ can be replaced by $\mathrm{H}_{1}\left(-, \mathbb{Z}_{\ell}\right)$.

Briefly, this proposition is proven by applying the Kummer exact sequence to obtain $\mathrm{H}^{1}\left((-)_{\bar{k}}, \mathbb{Z} / \ell^{n}(1)\right) \cong \operatorname{Pic}(-)_{\bar{k}}\left[\ell^{n}\right]$, and then relating torsion points and $\pi_{1}^{\ell}$ for algebraic groups.

Let $k$ and $\ell$ be as above. Let $p$ denote the characteristic of $k$, which could be 0 . $\mathrm{H}^{*}$ denotes étale cohomology. By an algebraic group, we mean a connected, smooth $k$-group scheme. An algebraic group is automatically geometrically connected by [10, Exposé $\mathrm{VI}_{\mathrm{A}}$, Proposition 2.4, page 299].

Lemma A.2 Let $G$ be a commutative algebraic group over $k$, and $N$ an integer which is prime to $p$. Then the $\bar{k}$-points of $G$ form an $N$-divisible group.

Proof The subcategory of groups whose $\bar{k}$-points are $N$-divisible is closed under extensions. The $\bar{k}$-points of $\mathbb{G}_{m}, \mathbb{G}_{a}$ and all abelian varieties are $N$-divisible. Thus the lemma follows by the classification of (connected) commutative, algebraic groups [11, Exposé XVII, Théorème 7.2.1, page 613].

Let $\pi_{1}^{\left(p^{\prime}\right)}$ denote the prime-to- $p$ étale fundamental group, and $\pi_{1}^{\ell}$ denote its maximal pro- $\ell$ quotient as above. By [8, Proposition 1.1 and Remark 4.3], it follows that:

Proposition A.3 If $G$ is a commutative algebraic group over $k$, then there is a natural isomorphism $\pi_{1}^{\left(p^{\prime}\right)}\left(G_{\bar{k}}, e\right) \cong \lim G[N](\bar{k})$, where $G[N]$ denotes the $N$-torsion and $N$ runs over positive integers which are prime to $p$.

Proof By [8, Proposition 1.1], $\pi_{1}^{\left(p^{\prime}\right)}\left(G_{\bar{k}}, e\right)$ is abelian, whence isomorphic to the maximal prime-to- $p$ quotient of $\lim _{Y} \operatorname{Gal}\left(Y / G_{\bar{k}}\right)$, where $Y$ runs over the finite, étale, abelian Galois covers of $G_{\bar{k}}$. Thus

$$
\pi_{1}^{\left(p^{\prime}\right)}\left(G_{\bar{k}}, e\right) \cong \underset{Y}{\lim } \operatorname{Gal}\left(Y / G_{\bar{k}}\right)^{\left(p^{\prime}\right)},
$$

where $\operatorname{Gal}\left(Y / G_{\bar{k}}\right)^{\left(p^{\prime}\right)}$ denotes the maximal prime-to- $p$ quotient of $\operatorname{Gal}\left(Y / G_{\bar{k}}\right)$. The quotient $\operatorname{Gal}\left(Y / G_{\bar{k}}\right)^{\left(p^{\prime}\right)}$ is naturally isomorphic to the Galois group of a finite, étale, 
abelian cover $Z_{Y} \rightarrow G_{\bar{k}}$ whose degree is prime to $p$. It follows that $\pi_{1}^{\left(p^{\prime}\right)}\left(G_{\bar{k}}, e\right) \cong$ $\lim _{Z} \operatorname{Gal}\left(Z / G_{\bar{k}}\right)$, where $Z$ runs over such covers. By [8, Remark 4.3], for $N$ prime to $p$,

$$
0 \rightarrow G_{\bar{k}}[N] \rightarrow G_{\bar{k}} \rightarrow G_{\bar{k}} \rightarrow 0
$$

is the largest abelian, étale, Galois cover of $G$ of exponent $N$, whence

$$
\pi_{1}^{\left(p^{\prime}\right)}\left(G_{\bar{k}}, e\right) \cong \lim G[N](\bar{k})
$$

as claimed.

Let $Z$ be a proper scheme over $k$. By [7, Section 8.2, Theorem 3], Pic $Z$ is represented by a locally finite type scheme over $k$, which is automatically a commutative group object. Let $\operatorname{Pic}^{0} Z$ denote the connected component of the identity $e$ of Pic $Z$, and $\left(\mathrm{Pic}^{0} Z\right)_{\text {red }}$ denote the reduced closed subscheme. By [18, Proposition 4.6.1, page 68], $\left(\mathrm{Pic}^{0} Z\right)_{\text {red }} \times_{k}\left(\mathrm{Pic}^{0} Z\right)_{\text {red }}$ is reduced, and it follows that $\left(\mathrm{Pic}^{0}\right)_{\text {red }}$ is a commutative group scheme. By generic smoothness, $\left(\mathrm{Pic}^{0}\right)_{\text {red }}$ is an algebraic group.

For $Z$ as above, let NS $Z_{\bar{k}}$ denote the Néron-Severi group of connected components of Pic $Z_{\bar{k}}$ [7, page 234],

$$
\mathrm{NS} Z_{\bar{k}}=\operatorname{Pic} Z_{\bar{k}}(\bar{k}) / \operatorname{Pic}^{0} Z_{\bar{k}}(\bar{k}) \cong \operatorname{Pic} Z(\bar{k}) / \operatorname{Pic}^{0} Z(\bar{k}) .
$$

Proposition A.4 For $N$ prime to $p$, there is a short exact sequence of functors

$$
0 \rightarrow \operatorname{Pic}^{0}(-)_{\bar{k}}[N](\bar{k}) \rightarrow \mathrm{H}^{1}\left((-)_{\bar{k}}, \mathbb{Z} / N(1)\right) \rightarrow \mathrm{NS}(-)_{\bar{k}}[N] \rightarrow 0
$$

from proper, geometrically connected schemes over $k$ of locally finite type to $\mathbb{Z} / N$ modules with $\operatorname{Gal}(\bar{k} / k)$-action.

Proof The Kummer exact sequence

$$
1 \rightarrow \mu_{N} \rightarrow \mathbb{G}_{m} \rightarrow \mathbb{G}_{m} \rightarrow 1
$$

and the fact that $\bar{k}^{*}$ is $N$-divisible gives an identification

$$
\mathrm{H}^{1}\left((-)_{\bar{k}}, \mathbb{Z} / N(1)\right)=\mathrm{H}^{1}\left((-)_{\bar{k}}, \mu_{N}\right) \cong \mathrm{H}^{1}\left((-)_{\bar{k}}, \mathbb{G}_{m}\right)[N] .
$$

By the definition of Pic, its $N$-torsion points over $\bar{k}$ are

$$
\operatorname{Pic}(-)_{\bar{k}}[N](\bar{k})=\mathrm{H}^{1}\left((-)_{\bar{k}}, \mathbb{G}_{m}\right)[N]
$$

Thus the claim is equivalent to showing a natural exact sequence

$$
0 \rightarrow \operatorname{Pic}^{0}(-)_{\bar{k}}[N](\bar{k}) \rightarrow \operatorname{Pic}(-)_{\bar{k}}[N](\bar{k}) \rightarrow \mathrm{NS}(-)_{\bar{k}}[N] \rightarrow 0 .
$$


By the definition of the Néron-Severi group

$$
0 \rightarrow \operatorname{Pic}^{0}(-)_{\bar{k}}(\bar{k}) \rightarrow \operatorname{Pic}(-)_{\bar{k}}(\bar{k}) \rightarrow \mathrm{NS}(-)_{\bar{k}} \rightarrow 0
$$

is exact, and it follows that

$$
\begin{aligned}
0 \rightarrow \operatorname{Pic}^{0}(-)_{\bar{k}}[N](\bar{k}) & \rightarrow \operatorname{Pic}(-)_{\bar{k}}[N](\bar{k}) \\
& \rightarrow \mathrm{NS}(-)_{\bar{k}}[N] \rightarrow \operatorname{Pic}^{0}(-)_{\bar{k}}(\bar{k}) / N \operatorname{Pic}^{0}(-)_{\bar{k}}(\bar{k})
\end{aligned}
$$

is also exact.

Note the canonical isomorphism $\operatorname{Pic}^{0}(-)_{\bar{k}}(\bar{k}) \cong\left(\operatorname{Pic}^{0}(-)_{\bar{k}}\right)_{\text {red }}(\bar{k})$. By the above, $\left(\mathrm{Pic}^{0}(-)_{\bar{k}}\right)_{\text {red }}$ is an algebraic group, so by Lemma A.2,

$$
\left(\operatorname{Pic}^{0}(-)_{\bar{k}}\right)_{\mathrm{red}}(\bar{k}) / N\left(\operatorname{Pic}^{0}(-)_{\bar{k}}\right)_{\mathrm{red}}(\bar{k}) \cong 0,
$$

proving the claim.

Proof of Proposition 1.8 By Proposition A.3, we have a natural isomorphism of functors

$$
\pi_{1}^{\ell}(-, e) \circ \operatorname{Pic}^{0}(-)_{\bar{k}}=\underset{N}{\lim } \operatorname{Pic}^{0}(-)_{\bar{k}}[N](\bar{k})
$$

where $N$ ranges over the powers of $\ell$, because the $\bar{k}$-points and étale fundamental group of $\mathrm{Pic}^{0}(-)_{\bar{k}}$ can be identified with those of its reduction, and $\left(\mathrm{Pic}^{0}(-)_{\bar{k}}\right)_{\text {red }}$ is a commutative algebraic group.

By Proposition A.4, we have exact sequences

$$
0 \rightarrow \mathrm{Pic}^{0}(-)_{\bar{k}}[N] \rightarrow \mathrm{H}^{1}\left((-)_{\bar{k}}, \mathbb{Z} / N(1)\right) \rightarrow \mathrm{NS}(-)_{\bar{k}}[N] \rightarrow 0,
$$

where $N$ ranges over the powers of $\ell$.

Taking the inverse limit gives the exact sequence

$$
\begin{aligned}
& 0 \longrightarrow \pi_{1}^{\ell}\left(\operatorname{Pic}^{0}(-)_{\bar{k}}, e\right) \cong \lim _{N} \operatorname{Pic}^{0}(-)_{\bar{k}}[N] \\
& \downarrow \\
& \mathrm{H}^{1}\left((-)_{\bar{k}}, \mathbb{Z}_{\ell}(1)\right):=\lim _{N} \mathrm{H}^{1}\left((-)_{\bar{k}}, \mathbb{Z} / N(1)\right) \longrightarrow \varliminf_{N} \mathrm{NS}(-)_{\bar{k}}[N] .
\end{aligned}
$$

Since NS $(-)_{\bar{k}}$ is finitely generated [7, Chapter 8.4, Theorem 7], multiplication by $\ell^{n}$ is the 0 map for large enough $n$, and if follows that $\lim _{N} \mathrm{NS}(-)_{\bar{k}}[N]=0$. 


\section{Appendix B: A Mayer-Vietoris sequence for pushouts of schemes}

Consider diagrams of finite-type schemes over $k$ of the form

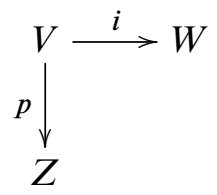

with $i$ a closed immersion and $p$ finite. By [15, Theorem 5.4], the pushout

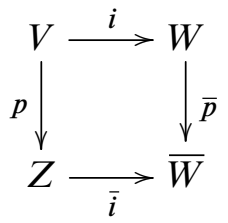

exists and commutes with base change by field extensions [15, Lemma 4.4]. The resulting morphisms $\bar{p}$ and $\bar{i}$ are finite, with $\bar{i}$ a closed immersion [15, Theorem 5.4]. This appendix proves a Mayer-Vietoris sequence in cohomology for pushouts of this form, and then obtains a truncation of such a sequence in homology. The truncation results from the fact that we only define $\mathrm{H}_{1}$ and $\mathrm{H}_{0}$. It is not mathematically essential.

Theorem B.1 There is a functorial association of a long exact sequence of $\operatorname{Gal}(\bar{k} / k)$ modules

$\cdots \rightarrow \mathrm{H}^{n}\left(\bar{W}_{\bar{k}}, R\right) \rightarrow \mathrm{H}^{n}\left(W_{\bar{k}}, R\right) \oplus \mathrm{H}^{n}\left(Z_{\bar{k}}, R\right) \rightarrow \mathrm{H}^{n}\left(V_{\bar{k}}, R\right) \rightarrow \mathrm{H}^{n+1}\left(\bar{W}_{\bar{k}}, R\right) \rightarrow \cdots$

to a pushout diagram (37).

Here the adjective "functorial" means that a map of pushout diagrams of the form (37) induces a map of long exact sequences.

Proof Let $f: V \rightarrow \bar{W}$ be defined by $f=\bar{i} p=\bar{p} i$. Let $R\left(V_{\bar{k}}\right)$ denote the constant sheaf with stalk $R$ on the étale site of $V_{\bar{k}}$ for $R=\mathbb{Z} / \ell^{m}$ and the corresponding $\ell$-adic sheaf for $R=\mathbb{Z}_{\ell}$ [25, page 163], with similar definitions for $W, Z$, and $\bar{W}$. Note that $\bar{p}^{*} R\left(\bar{W}_{\bar{k}}\right) \cong R\left(W_{\bar{k}}\right)$, giving a natural map $R\left(\bar{W}_{\bar{k}}\right) \rightarrow \bar{p}_{*} R\left(W_{\bar{k}}\right)$.

The horizontal arrows in the commutative diagram

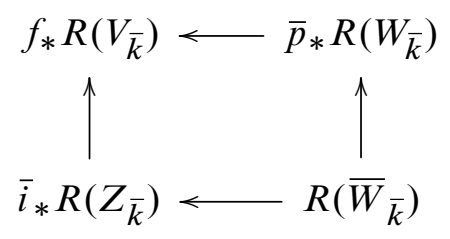


are surjective. Indeed, to check surjectivity, it is enough to verify the condition on stalks [25, Chapter II, Theorem 2.15(c)], and surjectivity on stalks follows from [25, Chapter II, Corollary 3.5(a)]. The resulting morphism of short exact sequences

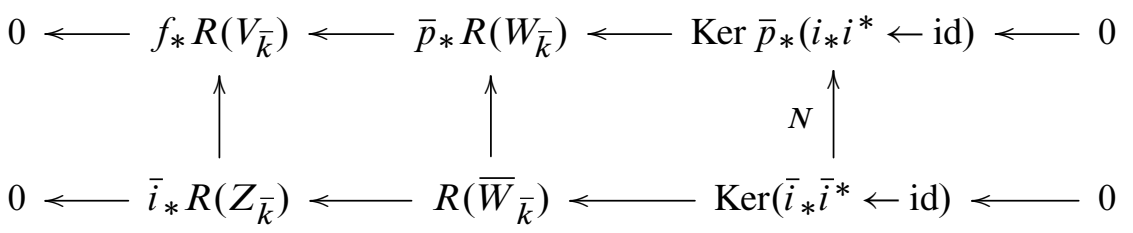

is such that $N$ is an isomorphism. To see this, note that by [25, Chapter II, Theorem 2.15(b) and Corollary 3.5(c)] pushforward by a finite morphism is an exact functor between categories of étale sheaves, whence $\operatorname{Ker} \bar{p}_{*}\left(i_{*} i^{*} \leftarrow \mathrm{id}\right)=\bar{p}_{*} \operatorname{Ker}\left(i_{*} i^{*} \leftarrow \mathrm{id}\right)$. Let $j: W-V \hookrightarrow W$ be the open immersion corresponding to the complement of $V$. The exact sequence [25, Chapter II, page 76]

$$
0 \rightarrow j ! j^{*} R \rightarrow R \rightarrow i_{*} i^{*} R \rightarrow 0
$$

gives $\operatorname{Ker}\left(i_{*} i^{*} \leftarrow \mathrm{id}\right) \cong j_{!} R\left((W-V)_{\bar{k}}\right)$. Similarly, $\operatorname{Ker}\left(\bar{i}_{*} \bar{i}^{*} \leftarrow \mathrm{id}\right) \cong \bar{j} ! R\left((\bar{W}-Z)_{\bar{k}}\right)$, where $\bar{j}: \bar{W}-Z \hookrightarrow \bar{W}$ is the open immersion. Since $\bar{p}$ induces an isomorphism $W-V \rightarrow \bar{W}-Z$ [15, Theorem 5.4(d)] and $\bar{p}^{-1} Z \subset V$ [15, Theorem 5.4(b)], $N$ is an isomorphism by checking on all stalks.

A morphism of short exact sequences of sheaves with isomorphic kernels

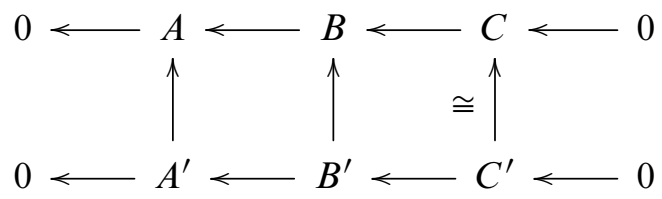

gives rise to a Mayer-Vietoris sequence

$$
\cdots \rightarrow \mathrm{H}^{n}\left(B^{\prime}\right) \rightarrow \mathrm{H}^{n}(B) \oplus \mathrm{H}^{n}\left(A^{\prime}\right) \rightarrow \mathrm{H}^{n}(A) \rightarrow \mathrm{H}^{n+1}\left(B^{\prime}\right) \rightarrow \cdots
$$

Applying this principle to (38) gives a long exact sequence

$$
\begin{aligned}
\cdots \rightarrow \mathrm{H}^{n}\left(\bar{W}_{\bar{k}}, R\right) \rightarrow \mathrm{H}^{n}\left(\bar{W}_{\bar{k}}, \bar{p}_{*} R\left(W_{\bar{k}}\right)\right) \oplus \mathrm{H}^{n}\left(\bar{W}_{\bar{k}}, \bar{i}_{*} R\left(Z_{\bar{k}}\right)\right) \\
\rightarrow \mathrm{H}^{n}\left(\bar{W}_{\bar{k}}, f_{*} R\left(V_{\bar{k}}\right)\right) \rightarrow \mathrm{H}^{n+1}\left(\bar{W}_{\bar{k}}, R\right) \rightarrow \cdots
\end{aligned}
$$

Since pushforward by a finite morphism is an exact functor between categories of étale sheaves, $\mathrm{H}^{n}\left(W_{\bar{k}}, R\right) \cong \mathrm{H}^{n}\left(\bar{W}_{\bar{k}}, \bar{p}_{*} R\left(W_{\bar{k}}\right)\right)$, and there are similar expressions for $\mathrm{H}^{n}\left(V_{\bar{k}}, R\right)$ and $\mathrm{H}^{n}\left(Z_{\bar{k}}, R\right)$. Making these substitutions into (39) completes the proof. 
Let $Y$ be a $k$-scheme. Let $\mathrm{H}_{0}\left(Y_{\bar{k}}, R\right)$ denote the free $R$-module on the connected components $\pi_{0}\left(Y_{\bar{k}}\right)$ of $Y_{\bar{k}}$. Since $\pi_{0}\left(Y_{\bar{k}}\right)$ has a continuous Galois action, $\mathrm{H}_{0}\left(Y_{\bar{k}}, R\right)$ is a $\operatorname{Gal}(\bar{k} / k)$-module. For $y \in \pi_{0}\left(Y_{\bar{k}}\right)$, let $\left(Y_{\bar{k}}\right)_{y}$ denote the corresponding connected component. For $R=\mathbb{Z} / \ell^{n}$ (or $R$ a finite abelian group), define $\mathrm{H}_{1}\left(Y_{\bar{k}}, R\right)$ as the direct sum

$$
\mathrm{H}_{1}\left(Y_{\bar{k}}, R\right) \cong \bigoplus_{y \in \pi_{0} Y_{\bar{k}}}\left(\pi_{1}\left(Y_{\bar{k}}\right)_{y}^{\mathrm{ab}} \otimes R\right)
$$

where $\pi_{1}\left(Y_{\bar{k}}\right)_{y}^{\mathrm{ab}}$ denotes the abelianization of the étale fundamental group of $\pi_{1}\left(Y_{\bar{k}}\right)_{y}$. For $R=\mathbb{Z}_{\ell}$, define $\mathrm{H}_{1}\left(Y_{\bar{k}}, \mathbb{Z}_{\ell}\right)$ as

$$
\mathrm{H}_{1}\left(Y_{\bar{k}}, \mathbb{Z}_{\ell}\right) \cong \bigoplus_{y \in \pi_{0} Y_{\bar{k}}} \pi_{1}\left(Y_{\bar{k}}\right)_{y}^{\ell-\mathrm{ab}}
$$

where $\pi_{1}\left(Y_{\bar{k}}\right)_{y}^{\ell-\mathrm{ab}}$ denotes the abelianization of the $\ell$-étale fundamental group, ie the abelianization of the maximal pro- $\ell$ quotient of the étale fundamental group. Note that $\pi_{1}\left(Y_{\bar{k}}\right)_{y}^{\mathrm{ab}}$ is independent of the choice of base point. The $\mathrm{Gal}(\bar{k} / k)$-action on étale paths in $Y_{\bar{k}}$ gives $\mathrm{H}_{1}\left(Y_{\bar{k}}, R\right)$ the structure of a $\operatorname{Gal}(\bar{k} / k)$-module. More specifically, for each $y \in \pi_{0}\left(Y_{\bar{k}}\right)$ choose a base point $b_{y}$, which may or may not have a $k$-rational point as its image, with which to define $\pi_{1}\left(\left(Y_{\bar{k}}\right)_{y}, b_{y}\right)$. Then $\sigma \in \mathrm{Gal}(\bar{k} / k)$ determines a map $\pi_{1}\left(\left(Y_{\bar{k}}\right)_{y}, b_{y}\right) \rightarrow \pi_{1}\left(Y_{\bar{k}}, \sigma_{*} b_{y}\right)$. Then $\sigma_{*} b_{y}$ has image in some connected component of $Y_{\bar{k}}$, say the connected component $w$. The canonical isomorphism

$$
\pi_{1}\left(\left(Y_{\bar{k}}\right)_{w}, b_{w}\right)^{\mathrm{ab}} \cong \pi_{1}\left(\left(Y_{\bar{k}}\right)_{w}, \sigma_{*} b\right)^{\mathrm{ab}}
$$

allows $\sigma$ to act on $\mathrm{H}_{1}\left(Y_{\bar{k}}, R\right)$.

We prove the following technical lemma to avoid considering the question of finite generation for $\pi_{1}^{\ell}$. Note that $\mathrm{H}_{1}\left(Y_{\bar{k}}, \mathbb{Z} / \ell^{m}\right)$ has a natural topology induced from the topology on étale fundamental groupoids. For a topological $\mathbb{Z} / \ell^{m}$-module $M$, let $M^{\star}=\operatorname{Hom}\left(M, \mathbb{Z} / \ell^{m}\right)$ be the continuous homomorphisms $M \rightarrow \mathbb{Z} / \ell^{m}$.

Lemma B.2 For each $y \in \pi_{0}\left(Y_{\bar{k}}\right)$, let $M_{y} \subseteq \mathrm{H}_{1}\left(\left(Y_{\bar{k}}\right)_{y}, \mathbb{Z} / \ell^{m}\right)$ be a closed sub$\mathbb{Z} / \ell^{m}$-module, and let $M=\bigoplus_{y} M_{y}$. For any $\gamma$ in $\mathrm{H}_{1}\left(Y_{\bar{k}}, \mathbb{Z} / \ell^{m}\right)$ with $\gamma \notin M$, there exists $f \in \mathrm{H}_{1}\left(Y_{\bar{k}}, \mathbb{Z} / \ell^{m}\right)^{\star}$ such that $f(\gamma) \neq 0$ and $f(m)=0$ for all $m \in M$.

Proof We may assume $k=\bar{k}$. By definition,

$$
\mathrm{H}_{1}\left(Y, \mathbb{Z} / \ell^{m}\right)=\bigoplus_{y \in \pi_{0}(Y)}\left(\pi_{1}\left(Y_{y}\right)^{\mathrm{ab}} \otimes \mathbb{Z} / \ell^{m}\right) .
$$


Since $\gamma$ is not in $M$ and $M=\bigoplus_{y} M_{y}$, there exists $y \in \pi_{0}(Y)$ such that the image $\gamma_{y}$ of $\gamma$ under

$$
\mathrm{H}_{1}\left(Y, \mathbb{Z} / \ell^{m}\right) \rightarrow \prod_{y \in \pi_{0}(Y)}\left(\pi_{1}\left(Y_{y}\right)^{\mathrm{ab}} \otimes \mathbb{Z} / \ell^{m}\right) \rightarrow \pi_{1}\left(Y_{y}\right)^{\mathrm{ab}} \otimes \mathbb{Z} / \ell^{m}
$$

is not contained in $M_{y}$. Using the map $\left(\pi_{1}\left(Y_{y}\right)^{\mathrm{ab}} \otimes \mathbb{Z} / \ell^{m}\right)^{\star} \rightarrow \mathrm{H}_{1}\left(Y_{\bar{k}}, \mathbb{Z} / \ell^{m}\right)^{\star}$, we may assume that $Y$ is connected. Since $\pi_{1}(Y)^{\mathrm{ab}} \otimes \mathbb{Z} / \ell^{m}$ is profinite, there is a finite quotient such that the image of $\gamma$ is not contained in the image of $M$, as otherwise the intersection over all finite quotients of those elements of $M$ with the same image as $\gamma$ would be non-empty by compactness of $M$, which in turn would imply that $\gamma$ is in $M$. Thus there exists an abelian finite étale cover $\bar{Y} \rightarrow Y$ such that the image of $\gamma$ under

$$
\mathrm{H}_{1}\left(Y, \mathbb{Z} / \ell^{m}\right) \rightarrow \operatorname{Gal}(\bar{Y} / Y) \otimes \mathbb{Z} / \ell^{m}
$$

is not contained in the image of $M$. The $\mathbb{Z} / \ell^{m}$-module $\left(\operatorname{Gal}(\bar{Y} / Y) \otimes \mathbb{Z} / \ell^{m}\right) / M$ is finitely generated (even finite), whence the natural map

$$
\left(\operatorname{Gal}(\bar{Y} / Y) \otimes \mathbb{Z} / \ell^{m}\right) / M \rightarrow\left(\left(\left(\operatorname{Gal}(\bar{Y} / Y) \otimes \mathbb{Z} / \ell^{m}\right) / M\right)^{\star}\right)^{\star}
$$

is an isomorphism. In particular, we may choose $\bar{f} \in\left(\left(\mathrm{Gal}(\bar{Y} / Y) \otimes \mathbb{Z} / \ell^{m}\right) / M\right)^{\star}$ such that $\bar{f}(\gamma) \neq 0$. Let $f$ be the image of $\bar{f}$ under the map

$$
\left(\left(\operatorname{Gal}(\bar{Y} / Y) \otimes \mathbb{Z} / \ell^{m}\right) / M\right)^{\star} \rightarrow\left(\operatorname{Gal}(\bar{Y} / Y) \otimes \mathbb{Z} / \ell^{m}\right)^{\star} \rightarrow \mathrm{H}_{1}\left(Y_{\bar{k}}, \mathbb{Z} / \ell^{m}\right)^{\star} .
$$

As a corollary, we obtain:

Lemma B.3 The natural map $\mathrm{H}_{1}\left(Y_{\bar{k}}, \mathbb{Z} / \ell^{m}\right) \rightarrow\left(\mathrm{H}_{1}\left(Y_{\bar{k}}, \mathbb{Z} / \ell^{m}\right)^{\star}\right)^{\star}$ is injective.

We use these lemmas to show the Mayer-Vietoris sequence in homology used above. Assume that $V_{\bar{k}}, Z_{\bar{k}}, W_{\bar{k}}$ and $\bar{W}_{\bar{k}}$ have finitely many connected components.

Theorem B.4 There is a functorial exact sequence of $\mathrm{Gal}(\bar{k} / k)$-modules

$$
\begin{aligned}
& \mathrm{H}_{1}\left(V_{\bar{k}}, R\right) \rightarrow \mathrm{H}_{1}\left(W_{\bar{k}}, R\right) \oplus \mathrm{H}_{1}\left(Z_{\bar{k}}, R\right) \rightarrow \mathrm{H}_{1}\left(\bar{W}_{\bar{k}}, R\right) \\
& \quad \rightarrow \mathrm{H}_{0}\left(V_{\bar{k}}, R\right) \rightarrow \mathrm{H}_{0}\left(W_{\bar{k}}, R\right) \oplus \mathrm{H}_{0}\left(Z_{\bar{k}}, R\right) \rightarrow \mathrm{H}_{0}\left(\bar{W}_{\bar{k}}, R\right) \rightarrow 0 .
\end{aligned}
$$

Proof Let $n=0,1$. Functoriality of $\mathrm{H}_{n}$ defines maps

$$
\begin{aligned}
\mathrm{H}_{n}(i) \times \mathrm{H}_{n}(p): \mathrm{H}_{n}\left(V_{\bar{k}}, R\right) & \rightarrow \mathrm{H}_{n}\left(W_{\bar{k}}, R\right) \oplus \mathrm{H}_{n}\left(Z_{\bar{k}}, R\right), \\
\mathrm{H}_{n}(i) \times \mathrm{H}_{n}(p)(x) & =\mathrm{H}_{n}(i) x \oplus \mathrm{H}_{n}(p) x
\end{aligned}
$$


and

$$
\begin{aligned}
-\mathrm{H}_{n}(\bar{p}) \oplus \mathrm{H}_{n}(\bar{i}): \mathrm{H}_{n}\left(W_{\bar{k}}, R\right) \oplus \mathrm{H}_{n}\left(Z_{\bar{k}}, R\right) & \rightarrow \mathrm{H}_{n}\left(\bar{W}_{\bar{k}}, R\right), \\
-\mathrm{H}_{n}(\bar{p}) \oplus \mathrm{H}_{n}(\bar{i})(x) & =-\mathrm{H}_{n}(\bar{p})(x)+\mathrm{H}_{n}(\bar{i})(x) .
\end{aligned}
$$

Suppose first that $R=\mathbb{Z} / \ell^{m}$. The isomorphism $\mathrm{H}^{1}\left(\left(Y_{\bar{k}}\right)_{y}, R\right) \cong \operatorname{Hom}\left(\pi_{1}\left(Y_{\bar{k}}\right)_{y}, R\right)$ between the first étale cohomology group and continuous homomorphisms from the fundamental group (this isomorphism can be obtained from, for instance, [25, Chapter III, Section 4; Section 2, Corollary 2.10 and Chapter I, Section 5]) determines an isomorphism

$$
\mathrm{H}^{1}\left(Y_{\bar{k}}, R\right) \cong \operatorname{Hom}\left(\mathrm{H}_{1}\left(Y_{\bar{k}}, R\right), R\right),
$$

where Hom again denotes continuous homomorphisms.

The isomorphism (41) determines a map $\mathrm{H}_{1}\left(\bar{W}_{k}, R\right) \rightarrow \mathrm{H}^{1}\left(\bar{W}_{\bar{k}}, R\right)^{\star}$. Dualizing the boundary map in Theorem B.1 gives a map

$$
\mathrm{H}^{1}\left(\bar{W}_{\bar{k}}, R\right)^{\star} \rightarrow \mathrm{H}^{0}\left(\bar{W}_{\bar{k}}, R\right)^{\star} .
$$

By assumption, $\bar{W}_{\bar{k}}$ has finitely many connected components, identifying $\prod_{\pi_{0} \bar{W}_{\bar{k}}} R$ and $\bigoplus_{\pi_{0} \bar{W}_{\bar{k}}} R$, and giving an isomorphism

$$
\mathrm{H}^{0}\left(\bar{W}_{\bar{k}}, R\right)^{\star} \cong \mathrm{H}_{0}\left(\bar{W}_{\bar{k}}, R\right) .
$$

Use the resulting composite

$$
\mathrm{H}_{1}\left(\bar{W}_{k}, R\right) \rightarrow \mathrm{H}^{1}\left(\bar{W}_{\bar{k}}, R\right)^{\star} \rightarrow \mathrm{H}^{0}\left(\bar{W}_{\bar{k}}, R\right)^{\star} \cong \mathrm{H}_{0}\left(\bar{W}_{\bar{k}}, R\right)
$$

to define the boundary map in the statement we are proving. Together with $\mathrm{H}_{n}(i) \times$ $\mathrm{H}_{n}(p)$ and $-\mathrm{H}_{n}(\bar{p}) \oplus \mathrm{H}_{n}(\bar{i})$, this defines the sequence in the statement.

Note that, with this definition, applying $\operatorname{Hom}(-, R)$ to the sequence of Theorem B.4 gives the sequence of Theorem B.1. It thus follows from Lemma B.3 that the composition of adjacent maps in the sequence of Theorem B.4 is 0 , ie this sequence is a complex. We claim that this complex is exact. Suppose to the contrary that exactness fails at

$$
M_{j+1} \rightarrow M_{j} \rightarrow M_{j-1}
$$

ie there exists $\gamma$ in $\operatorname{Ker}\left(M_{j} \rightarrow M_{j-1}\right)$ which is not in $\operatorname{Image}\left(M_{j+1} \rightarrow M_{j}\right)$. Choose $f \in M_{j}^{\star}$ vanishing on the image of $M_{j+1}$ and nonzero on $\gamma$, using Lemma B.2. Then $f$ is in the kernel of $M_{j}^{\star} \rightarrow M_{j+1}^{\star}$. However, $f$ cannot be in the image of $M_{j-1}^{\star} \rightarrow M_{j}^{\star}$ because any element of this image sends $\gamma$ to 0 . 
The quotient map $\mathbb{Z} / \ell^{m+1} \rightarrow \mathbb{Z} / \ell^{m}$ determines maps

$$
\mathrm{H}_{n}\left(Y_{\bar{k}}, \mathbb{Z} / \ell^{m+1}\right) \rightarrow \mathrm{H}_{n}\left(Y_{\bar{k}}, \mathbb{Z} / \ell^{m}\right) .
$$

It is tautological to check that these maps induce maps between the corresponding exact sequences in the statement. The case $R=\mathbb{Z}_{\ell}$ then follows from the isomorphisms

$$
\mathrm{H}_{n}\left(Y_{\bar{k}}, \mathbb{Z}_{\ell}\right) \cong \underset{m}{\lim } \mathrm{H}_{n}\left(Y_{\bar{k}}, \mathbb{Z} / \ell^{m}\right)
$$

and exactness of inverse limits on profinite groups.

\section{References}

[1] A B Altman, S L Kleiman, Compactifying the Picard scheme, Adv. in Math. 35 (1980) 50-112 MR555258

[2] A B Altman, S L Kleiman, The presentation functor and the compactified Jacobian, from: "The Grothendieck Festschrift, Vol. I", (P Cartier, L Illusie, N M Katz, G Laumon, Y Manin, K A Ribet, editors), Progr. Math. 86, Birkhäuser, Boston, MA (1990) 15-32 MR1086881

[3] D Arinkin, Cohomology of line bundles on compactified Jacobians, Math. Res. Lett. 18 (2011) 1215-1226

[4] D Arinkin, Autoduality of compactified Jacobians for curves with plane singularities, J. Algebraic Geom. 22 (2013) 363-388

[5] MF Atiyah, Thom complexes, Proceedings London Math. Soc. 11 (1961) 291-310 MR0131880

[6] U Bhosle, Generalized parabolic bundles and applications to torsionfree sheaves on nodal curves, Ark. 30 (1992) 187-215

[7] S Bosch, W Lütkebohmert, M Raynaud, Néron models, Ergeb. Math. Grenzgeb. 21, Springer, Berlin (1990) MR1045822

[8] M Brion, T Szamuely, Prime-to- $p$ étale covers of algebraic groups and homogeneous spaces, Bull. Lond. Math. Soc. 45 (2013) 602-612

[9] P Deligne, Cohomologie étale (SGA $4 \frac{1}{2}$ ), Lecture Notes in Mathematics 569, Springer, Berlin (1977) MR0463174

[10] M Demazure, A Grothendieck (editors), Schémas en groupes, I: Propriétés générales des schémas en groupes (SGA 3), Lecture Notes in Math. 151, Springer, Berlin (1970) MR0274458

[11] M Demazure, A Grothendieck (editors), Schémas en groupes, II: Groupes de type multiplicatif, et structure des schémas en groupes généraux (SGA 3), Lecture Notes in Math. 152, Springer, Berlin (1970) MR0274459 
[12] E Esteves, M Gagné, S L Kleiman, Abel maps and presentation schemes, Comm. Algebra 28 (2000) 5961-5992

[13] E Esteves, M Gagné, S L Kleiman, Autoduality of the compactified Jacobian, J. London Math. Soc. 65 (2002) 591-610 MR1895735

[14] E Esteves, F Rocha, Autoduality for treelike curves with planar singularities, Bull. Braz. Math. Soc. 44 (2013) 413-420

[15] D Ferrand, Conducteur, descente et pincement, Bull. Soc. Math. France 131 (2003) 553-585 MR2044495

[16] EM Friedlander, Étale homotopy of simplicial schemes, Annals of Mathematics Studies 104, Princeton Univ. Press (1982) MR676809

[17] A Grothendieck, Revêtements étales et groupe fondamental: Séminaire de géométrie algébrique du Bois Marie 1960-61 (SGA 1), Lecture Notes in Math. 224, Springer, Berlin (1962) MR2017446 Reprinted by Soc. Math. France, Paris, 2003

[18] A Grothendieck, J Dieudonné, Eléments de géométrie algébrique, IV: Étude locale des schémas et des morphismes de schémas, II, Inst. Hautes Études Sci. Publ. Math. 24 (1965) 5-231

[19] P Hu, On the Picard group of the stable $\mathbb{A}^{1}$-homotopy category, Topology 44 (2005) 609-640 MR2122218

[20] D C Isaksen, Etale realization on the $\mathbb{A}^{1}$-homotopy theory of schemes, Adv. Math. 184 (2004) 37-63 MR2047848

[21] J L Kass, An explicit non-smoothable component of the compactified Jacobian, J. Algebra 370 (2012) 326-343

[22] J L Kass, Singular curves and their compactified Jacobians, from: "A celebration of algebraic geometry”, (B Hassett, J McKernan, J Starr, R Vakil, editors), Clay Math. Proc. 18, Amer. Math. Soc. (2013) 391-427

[23] H Kleppe, S L Kleiman, Reducibility of the compactified Jacobian, Compositio Math. 43 (1981) 277-280 MR622452

[24] M Melo, A Rapagnetta, F Viviani, Fourier-Mukai and autoduality for compactified Jacobians, I arXiv:1207.7233

[25] J S Milne, Étale cohomology, Princeton Mathematical Series 33, Princeton Univ. Press (1980) MR559531

[26] S Mochizuki, Topics in absolute anabelian geometry, I: Generalities, J. Math. Sci. Univ. Tokyo 19 (2012) 139-242

[27] D Mumford, Abelian varieties, Tata Inst. Fund. Res. Studies Math. 5, Tata Institute, Bombay (1970) MR0282985

[28] D Mumford, Curves and their Jacobians, The University of Michigan Press, Ann Arbor, Mich. (1975) MR0419430 
[29] T Oda, C S Seshadri, Compactifications of the generalized Jacobian variety, Trans. Amer. Math. Soc. 253 (1979) 1-90 MR536936

Department of Mathematics, University of South Carolina 1523 Greene Street, Columbia, SC 29208, USA

School of Mathematics, Georgia Institute of Technology 686 Cherry Street, Atlanta, GA 30332, USA

kassj@math.sc.edu, wickelgren@post.harvard.edu http://www2.iag. uni-hannover.de/ kass/, http://people.math.gatech.edu/ kwickelgren3/

Received: 15 January 2014 Revised: 24 June 2014 
